\title{
Sensitivity of steady states in a degenerately-damped stochastic Lorenz system
}

\author{
Juraj Földes, Nathan E. Glatt-Holtz, David P. Herzog \\ emails: foldes@virginia.edu, negh@tulane.edu, dherzog@iastate.edu
}

\begin{abstract}
We study stability of solutions for a randomly driven and degenerately damped version of the Lorenz '63 model. Specifically, we prove that when damping is absent in one of the temperature components, the system possesses a unique invariant probability measure if and only if noise acts on the convection variable. On the other hand, if there is a positive growth term on the vertical temperature profile, we prove that there is no normalizable invariant state. Our approach relies on the derivation and analysis of non-trivial Lyapunov functions which ensure positive recurrence or nullrecurrence/transience of the dynamics.
\end{abstract}

Keywords: Stochastic Differential Equations, The Lorenz '63 System, Invariant Measures, Noise Induced Stabilization, Lyapunov Construction

MSC2020: 37Н30, 37А25, 37А30, 37A50

\section{Contents}

1. Introduction

2. Methodological Foundations: Lyapunov Techniques

3. Non-explosivity and Uniqueness Results

4. Positive Recurrence in the Absence of Damping

5. Sensitivity with Respect to Convective Forcing.

6. Non-Existence of Stationary States in the Presence of a Linear Instability 20

Acknowledgements 


\section{INTRODUCTION}

In this manuscript, we study the existence of invariant measures of the stochastic Lorenz system

$$
\begin{aligned}
& d x=\sigma(y-x) d t+\sqrt{2 \gamma_{1}} d B_{1}, \\
& d y=x(\rho-z) d t-y d t+\sqrt{2 \gamma_{2}} d B_{2}, \\
& d z=x y d t-\beta z d t+\sqrt{2 \gamma_{3}} d B_{3},
\end{aligned}
$$

where $B_{i}, i=1,2,3$ are independent, standard Brownian motions and $\sigma, \rho, \beta, \gamma_{i}$ are constants. We assume that $\sigma>0$ and $\rho \geq 0$, while for the diffusion parameters $\gamma_{1}, \gamma_{2}, \gamma_{3} \geq 0$ we require $\gamma_{i}>0$ for at least one index $i$, which means that the system is genuinely stochastic. If $\beta>0$, it is well known that (1.1) possesses a normalizable invariant measure and the long term dynamics has been extensively studied. In the present paper we focus on a 'degenerate damping factor' $\beta \leq 0$, and we investigate whether the presence of noise plays a nontrivial role in stabilizing the dynamics.

Previous Literature. The deterministic version of equation (1.1); that is, when $\gamma_{i}=0$ for $i=1,2,3$, has a long history as a canonical example of a chaotic dynamical system. Originally (1.1) was derived from the Boussinesq approximation of Rayleigh-Bénard convection, [Lor63]. It is understood as a projection of the Boussinesq equation onto one Fourier direction with wavenumber $k$, in which case $x$ represents the convection rate, and $y$ and $z$ describe the horizontal and vertical temperature variations, respectively. In this framing as a simple model for convection, $\sigma$ corresponds to the Prandtl number, $\rho$ is a rescaled Rayleigh number and $\beta$ is an aspect ratio depending on $k$.

While certainly $\beta$ is a strictly postive parameter in the original derivation of (1.1), if the Rayleigh number is large, a typical assumptions for turbulent flows, and $k$ is large, then $\beta \approx 0$. Thus it is natural to investigate the system with $\beta=0$. On the other hand, practical numerical considerations lead to the so-called Homogeneous Raylegh-Bénard (HRB) system, where a linearly unstable term, an analogue of the case when $\beta \leq 0$, appears in the temperature equation. See, for example, [CDG $\left.{ }^{+} 06\right]$ and a related two-dimensional ODE stochastic model in [BD12]. Furthermore, equations with similar structure to HRB also appear in a certain zero Prandlt limit which models mantle convection, see for example [Thu92, SS17]. Thus both HRB and the zero Prandlt limit provide additional motivation for studying the parameter range $\beta \leq 0$ in (1.1).

Here it is worth emphasizing that noise must be introducted in (1.1) for there to be any hope that this system would posses any (globally) stable statistics when $\beta \geq 0$. Indeed, in the absence of noise when $\beta<0$, the system (1.1) has initial conditions $\left(x_{0}=y_{0}=0, z_{0} \neq 0\right)$ leading to infinite time blow-up. On the other hand if $\beta=0$, then all points on $z$-axis are equilibria, and therefore there is no compact global attractor. Nevertheless, in both cases, the set of initial conditions that lead to blow-up (or equilibria) sit on a lower dimensional subset of the phase space. One may therefore inquire if one can find suitable noise perturbations which kick trajectories off of these meager subset of the phase space stabilizing the dynamics and leading formation of statistically steady states.

Thus the topics studied in this paper for $\beta \leq 0$ fall into a larger class of "stabilization-by-noise" problems. Such problems have been investigated by a number of researchers in a variety of contexts. Let us next briefly recall those works closely related to our setting. Motivated by convection models in [HP90a, HP90b], the effect of additive noise on unbounded solutions was studied. From another perspective advocated recently in [EHS17], the range $\beta \leq 0$ above provides a turbulence analogue of a class of core models in non-equilibrium statistical mechanics describing coupled oscillators with heat baths at different temperatures [RBT02, EH00, CEHRB18, Hai09, HM09]. Similar to such works on heat baths, one associates a natural energy functional with (1.1) which is "approximately conserved" but which is not globally dissipative. In particular, dissipation naturally acts on the $x$ and $y$ directions, but not necessarily on the $z$-direction, unless of course $\beta>0$. However, when $\beta \leq 0$, either there is no explicit dissipation $(\beta=0)$ or there is in fact a source of linear instability $(\beta<0)$, so it is unclear whether the dissipation in $x$ and $y$, coupled with the noise, can conspire to "spread" the dissipation to the $z$-direction. Let us finally mention that it is known that an arbitrary 
small additive noise can avert deterministic finite-time blow-up and lead to stable dynamics, see for example [Sch93, GHW11, BHW12, AKM12, HM15a, LS17, CFK $\left.{ }^{+} 17\right]$. The presence of noise can also induce stable oscillations [BD12] among other behaviors [KCSW19].

Statement of the Main Results. In view of the above discussions we aim to answer the following question in this paper:

Question 1.1. For what values of $\beta \leq 0$ and of $\gamma_{1}, \gamma_{2}, \gamma_{3} \geq 0$ does (1.1) possess an invariant probability measure?

Recall that in our context there is at least one index $i$ such that $\gamma_{i}>0$. The answer to this question is known to be affirmative for $\beta>0$ and, in fact, in this case the system is geometrically ergodic when $\gamma_{1}>0$ and either $\gamma_{2}>0$ or $\gamma_{3}>0$; see, for example, [MSH02]. Thus, our focus in this paper is on the case when $\beta \leq 0$, where the associated deterministic dynamics does not possess a compact global attractor.

Let us now present the main results in the paper concerning the stochastic stability of (1.1) which addresses most of Question 1.1.

Theorem 1.2. Consider (1.1) and assume $\sigma>0, \rho \geq 0$ and $\gamma_{1}, \gamma_{2}, \gamma_{2} \geq 0$ with at least one index $i$ for which $\gamma_{i}>0$. For any value of $\beta \leq 0$, the stochastic dynamics is globally defined (non-explosive in the sense of (2.4) below).

(i) If $\beta=0, \gamma_{1}>0$, then (1.1) has a unique invariant probability measure.

(ii) If $\beta=0, \gamma_{1}=0$, and one of $\gamma_{2}, \gamma_{3}$ is positive, then (1.1) does not possess an invariant probability measure.

(iii) Finally if $\beta<0$, then for any $\mathcal{K} \subseteq \mathbb{R}^{3}$ compact, there exists $(x, y, z) \notin \mathcal{K}$ such that

$$
\mathbb{E}_{(x, y, z)} \xi_{\mathcal{K}}=\infty
$$

where

$$
\xi_{\mathcal{K}}=\inf \left\{t \geq 0:\left(x_{t}, y_{t}, z_{t}\right) \in \mathcal{K}\right\} .
$$

Consequently, if we furthermore assume that $\gamma_{1}>0$ and either $\gamma_{2}>0$ or $\gamma_{3}>0$, then (1.1) does not possess an invariant probability measure.

Remark 1.3. Depending on which noise parameters $\gamma_{i}$ are positive, the issue of uniqueness of invariant measures for the system (1.1) can also be subtle. In the recent interesting paper [CZH20], it is shown that when $\beta>0, \gamma_{1}=\gamma_{2}=0$ and $\gamma_{3}>0$, then invariant measures can either be unique or not, and the uniqueness depends on the magnitude of the non-zero noise parameter $\gamma_{3}>0$.

Overview of the Analysis. Since the coefficients of equation (1.1) are globally smooth $\left(C^{\infty}\right)$ functions, the proof of well-posedness of (1.1) follows immediately once one establishes absence of finitetime explosion. In our case, non-explosivity is then concluded by using the natural Lyapunov function associated to the dynamics. See Proposition 3.1 for further details. However, since $\beta \leq 0$, this natural function is not robust enough to determine the existence/non-existence of an invariant probability measure precisely because of the absence of explicit dissipation in the $z$-direction. Thus we cannot use this function directly to answer our main question.

To this end, the typical route used to conclude existence/non-existence of an invariant probability measure is to estimate, for a 'big' compact set $\mathcal{K} \subseteq \mathbb{R}^{3}$, the expectation of the random variable $\xi_{\mathcal{K}}$ as in (1.2). Indeed, if one can show that $(x, y, z) \mapsto \mathbb{E}_{(x, y, z)} \xi_{\mathcal{K}}{ }^{1}$ is bounded on compact sets in $\mathbb{R}^{3}$, then an invariant probability measure can be constructed using a slight modification of the cycle argument of Khasminskii [Kha11]. See also [Bel06, $\mathrm{K}^{+} 87$ ]. On the other hand, if there is sufficient noise in the system (1.1) by way of hypoellipticity and support properties of the solution, then global finiteness of the function $\mathbb{E}_{(x, y, z)} \xi_{\mathcal{K}}$ for some $\mathcal{K}$ compact is equivalent to the existence of an invariant probability

\footnotetext{
$1_{\mathbb{E}_{(x, y, z)}}$ denotes the expectation with respect to the process $\left(x_{t}, y_{t}, z_{t}\right)$ started at $(x, y, z)$ at time 0
} 
measure $\left[\mathrm{K}^{+} 87\right]$. Thus our arguments center around determining whether or not this expectation can be shown to be globally finite.

Our approach to estimating $\mathbb{E}_{(x, y, z)} \xi_{\mathcal{K}}$ relies on detailed Lyapunov constructions. Specifically, to show the expectation is bounded on compact sets, we seek a $C^{2}$ function $V: \mathbb{R}^{3} \rightarrow \mathbb{R}$ with $V(x, y, z) \rightarrow$ $\infty$ as $|(x, y, z)| \rightarrow \infty$ and such that

$$
\mathcal{L} V \leq-c+d \mathbb{1}_{\mathcal{K}}
$$

for some $c, d>0$ and some compact set $\mathcal{K} \subset \mathbb{R}^{3}$, where $\mathcal{L}$ is the infinitesimal generator of (1.1) defined in (3.1). While the above Lyapunov criteria is quite standard to show existence of an invariant probability measure (see, for example, [Kha11, MT93, Bel06]), we will see that Lyapunov constructions can also be employed to establish nonexistence. Following [Won66] and generalizations more recently appearing in [Hai09, Kha11], Theorem 2.2 below identifies a condition guaranteeing infinite expected return times depending on the existence of two test functions, $V_{1}, V_{2}: \mathbb{R}^{3} \rightarrow \mathbb{R}$ satisfying Lyapunov-like conditions.

Regarding the existence of an invariant probability measure when $\beta=0$, we construct a suitable Lyapunov function $V$ satisfying (1.3) by pivoting off of the natural Lyapunov function one uses to show well-posedness, namely

$$
H(X)=H(x, y, z):=x^{2}+y^{2}+z^{2}-2(\sigma+\rho) z .
$$

Here, a direct computation, see (3.1), leads to

$$
\mathcal{L} H=-2\left(\sigma x^{2}+y^{2}\right)-2 \beta z^{2}+2 \beta(\sigma+\rho) z+2\left(\gamma_{1}+\gamma_{2}+\gamma_{3}\right)
$$

which reveals the necessity of adding supplemental terms to $H$ thus seeking a Lypunov function of the form $H+\psi$ in order to achieve (1.3) for the region where $x, y$ are bounded but where $|z|$ is large; that is (1.3) requires $\mathcal{L}(H+\psi)$ to be uniformly negative off of a compact set. The definition of the supplemental perturbation $\psi$ makes use of certain rescalings of the dynamics at large values, allowing one to better parse relevant terms in a neighborhood of the point at infinity. Note that these asymptotics initially yield a piecewise definition of the perturbation $\psi$ which, in order to obtain a globally $C^{2}$ requires that the different regions be glued together. The detailed derivation of $\psi$ and the motivation behind the scaling we choose are carried out in Section 4.

To extract conclusion (iii) in Theorem 1.2, we again employ Lyapunov methods in order to estimate the expected return time to a given compact set $\mathcal{K}$. The principal observation leading to the proof is that the function $M$ given by

$$
M(X)=M(x, y, z)=2 \sigma z-x^{2}
$$

satisfies

$$
\mathcal{L} M=2 \sigma\left(|\beta| z+x^{2}\right)-\gamma_{1} .
$$

Now, if it were the case (although it is far from true) that the set $\{(x, y, z): \sigma z \geq x\}$ is invariant for the dynamics, then (1.6) and (1.7) would together imply that the solution is growing exponentially in this region, provided $z>0$ is large enough initially. The proof then nontrivially modifies this initial observation to conclude the result, even in the presence of noise or dynamics effects that can steer the process in and out of this region. See Section 6 for further details.

Finally, to treat the borderline case $\beta=0$ when $\gamma_{1}=0$, we proceed with a direct approach. For example, when $\gamma_{1}=\beta=0$ but either $\gamma_{2}$ or $\gamma_{3}$ are strictly positive we again use of the test function $M$ defined in (1.6). At least formally, (1.7) and Dynkin's formula immediately implies that $\mathbb{E} x \equiv 0$ if the initial condition is distributed as an invariant state. However, this produces a contradiction to the structure of (1.1), since if $\gamma_{2}>0$, then $y$ is non-trivial, leading to non-zero derivative of $x$. On the other hand, if $\gamma_{3}>0$ and $y=0$, then $z$ evolves as a Brownian motion, which is not a normalizable invariant. Similar direct argumentation can be employed to show that when $\gamma_{2}=\gamma_{3}=\beta=0$ and $\gamma_{1}>0$, then the only stationary solution is an Ornstein-Uhlenbeck process concentrated on the $x$ component. 
Organization. The rest of the manuscript is organized as follows. Section 2 provides a self contained summary of some general results on Lyapunov methods for Itô diffusions. Section 3 contains the details for nonexplosivity and conditions for the uniqueness of invariant measures for (1.1). The results of this section also imply the uniqueness part of Theorem 1.2 (i) when $\gamma_{1}>0$ and either $\gamma_{2}>0$ or $\gamma_{3}>0$. In Section 4, we carry out the construction and rigorous analysis of a Lyapunov function leading to the existence of an invariant probability (1.1) in the case when $\beta=0$ and $\gamma_{1}>0$. The main result in this section, Proposition 4.1, in particular establishes the existence part of Theorem 1.2 (i). In Section 5, we prove Theorem 1.2 (ii) and establish uniqueness of the invariant measure when $\beta=0, \gamma_{1}>0$ and $\gamma_{2}=\gamma_{3}=0$, thus finalizing the proof of Theorem 1.2 (i). Finally, in Section 6 we prove Theorem 1.2 (iii). This proof also relies on Lyapunov constructions.

\section{Methodological Foundations: Lyapunov Techniques}

This section presents some general results on Lyapunov methods for Itô diffusions, providing the foundation for our analysis in later sections. To keep the paper self-contained, we present detailed proofs for some results familiar to specialists, but which are dispersed in literature under varied sets of assumptions.

Let $M_{n k}$ denote the set of $n \times k$ matrices with entries in $\mathbb{R}$. Given any $F \in C^{2}\left(\mathbb{R}^{n} ; \mathbb{R}^{n}\right)$ and $G=\left(G_{1}, \ldots, G_{k}\right) \in C^{2}\left(\mathbb{R}^{n} ; M_{n k}\right)$, let $X_{t}$ be the process on $\mathbb{R}^{n}$ satisfying the Itô stochastic differential equation

$$
d X_{t}=F\left(X_{t}\right) d t+G\left(X_{t}\right) d B_{t}=F\left(X_{t}\right) d t+\sum_{l=1}^{k} G_{l}\left(X_{t}\right) d B_{t}^{l}
$$

Here, $B_{t}=\left(B_{t}^{1}, \ldots, B_{t}^{k}\right)^{T}$ is a standard $k$-dimensional Brownian motion defined on a filtered probability space $\left(\Omega, \mathcal{F},\left\{\mathcal{F}_{t}\right\}_{t \geq 0}, \mathbb{P}\right)$, where $\mathbb{E}$ denotes the corresponding expected value. We denote by $\mathcal{L}$ the infinitesimal generator of the process $X_{t}$ acting on functions $V \in C^{2}\left(\mathbb{R}^{n} ; \mathbb{R}\right)$, namely,

$$
\begin{aligned}
\mathcal{L} V(X) & :=F(X) \cdot \nabla V(X)+\frac{1}{2}\left(G G^{T}\right)(X) \nabla^{2} V(X) \\
& =\sum_{j=1}^{n} F_{j}(X) \partial_{X_{j}} V(X)+\frac{1}{2} \sum_{i, j=1}^{n} \sum_{l=1}^{k} G_{i l} G_{j l}(X) \partial_{X^{i} X^{j}}^{2} V(X) .
\end{aligned}
$$

Let $\mathcal{B}$ be the Borel $\sigma$-field of subsets of $\mathbb{R}^{n}$.

Although globally defined solutions (2.1) in time are not guaranteed for general $C^{2}$ drifts $F$ and diffusions $G$, there are unique pathwise solutions defined until the first time $\tau$ in which the process leaves every bounded domain in space. Specifically, given a fixed initial condition $X$, if we define stopping times

$$
\tau_{n}:=\inf \left\{t \geq 0:\left|X_{t}\right| \geq n\right\}, \text { and } \tau:=\lim _{n \rightarrow \infty} \tau_{n},
$$

then solutions exist and are unique for all times $t<\tau, \mathbb{P}$-almost surely. We call $\tau$ the explosion time of the process $X_{t}$ and say that $X_{t}$ is nonexplosive if

$$
\mathbb{P}_{X}\{\tau<\infty\}=0 \text { for all initial conditions } X \in \mathbb{R}^{n} .
$$

In the above (2.4), the notation $\mathbb{P}_{X}$ means the process $X_{t}$ has $X_{0}=X \in \mathbb{R}^{n}$.

If $X_{t}$ is nonexplosive, solutions of (2.1) exist and are unique for all times $t \geq 0$ almost surely. Moreover, $X_{t}$ generates a Markov process and we define the transition probability measure $\mathcal{P}_{t}$ as $\mathcal{P}_{t}(X, \cdot)=\mathbb{P}_{X}\left\{X_{t} \in \cdot\right\}$. The Markov semigroup $\mathcal{P}_{t}$ acts on bounded, $\mathcal{B}$-measurable functions $V:$ $\mathbb{R}^{n} \rightarrow \mathbb{R}$ via

$$
\mathcal{P}_{t} V(X)=\mathbb{E}_{X} V\left(X_{t}\right)=\int_{\mathbb{R}^{n}} V(Y) \mathcal{P}_{t}(X, d Y), \quad X \in \mathbb{R}^{n},
$$


and on borel measures $\pi$ according to

$$
\pi \mathcal{P}_{t}(A)=\int_{\mathbb{R}^{n}} \pi(d X) \mathcal{P}_{t}(X, A) \quad A \in \mathcal{B}
$$

We say that a positive measure $\pi$ is an invariant measure for $\mathcal{P}_{t}$ if $\pi \mathcal{P}_{t}=\pi$ for all $t \geq 0$. An invariant measure $\pi$ for $\mathcal{P}_{t}$ with $\pi\left(\mathbb{R}^{n}\right)=1$ is called an invariant probability measure for $\mathcal{P}_{t}$.

The next result outlines the criteria for a process defined by (2.1) to be both nonexplosive and have finite expected returns to a compact set.

Proposition 2.1. Given $F, G \in C^{2}$, the following statements hold for solutions $X_{t}$ of (2.1) and the corresponding infinitesimal generator $\mathcal{L}$ defied in $(2.2)$.

(a) Suppose that there exist a function $V \in C^{2}\left(\mathbb{R}^{n} ; \mathbb{R}\right)$ such that $V(X) \rightarrow \infty$ as $|X| \rightarrow \infty$ and constants $c, d>0$ with the global bound

$$
\mathcal{L} V(X) \leq c V(X)+d \quad \text { for all } \quad X \in \mathbb{R}^{n} .
$$

Then $X_{t}$ is nonexplosive, namely (2.4) holds.

(b) Suppose that $X_{t}$ is nonexplosive and that there exist a function $V \in C^{2}\left(\mathbb{R}^{n} ;[0, \infty)\right)$, a compact set $\mathcal{K} \subseteq \mathbb{R}^{n}$ and constants $c, d>0$ such that

$$
\mathcal{L} V(X) \leq-c+d \mathbf{1}_{\mathcal{K}}(X) \quad \text { for all } \quad X \in \mathbb{R}^{n} .
$$

If

$$
\xi_{\mathcal{K}}:=\inf \left\{t \geq 0: X_{t} \in \mathcal{K}\right\}
$$

denotes the first hitting time of $\mathcal{K}$ by $X_{t}$, then

$$
\mathbb{E}_{X} \xi_{\mathcal{K}} \leq \frac{V(X)}{c},
$$

for all $X \in \mathbb{R}^{n}$.

The proof of the proposition above is a straightforward application of Itô's formula and can be found in a number of references, see, for example [Kha11, MT93, Bel06]. To illustrate the basic idea, we provide details for part (b).

Proof of Proposition 2.1 (b). Take $\xi:=\xi_{t, n, \mathcal{K}}:=t \wedge \tau_{n} \wedge \xi_{\mathcal{K}}$ with $\tau_{n}$ defined as in (2.3). If $X \in \mathcal{K}$, then $\mathbb{E}_{X} \xi_{\mathcal{K}}=0$ and (2.8) follows. Otherwise, $\mathbf{1}_{\mathcal{K}}(X)=0$ and by Dynkin's formula and (2.6) we have

$$
0 \leq \mathbb{E}_{X} V\left(X_{\xi}\right)=V(X)+\mathbb{E}_{X} \int_{0}^{\xi} \mathcal{L} V\left(X_{s}\right) d s \leq V(X)-c \mathbb{E}_{X} \xi
$$

for any $t \geq 0$ and $n \geq 1$. Rearranging and using $V \geq 0$ produces the estimate

$$
\mathbb{E}_{X} \xi_{t, n, \mathcal{K}} \leq \frac{V(X)}{c} .
$$

Passing $n \rightarrow \infty$ and then $t \rightarrow \infty$ using both monotone convergence and nonexplosivity, that is, $\tau_{n} \rightarrow \infty$ a.s., gives the desired bound (2.8).

The next result provides the criteria we use in Section 6 to show that the expected return time to compact sets is infinite for some initial data in the case case when the parameter $\beta<0$ in equation (1.1). While the result presented here can be traced back to at least [Won66] we believe it deserves further attention as a powerful tool for the study of stochastic (in)stability. Note that the original formulation in [Won66] imposes more hypotheses on the process $X_{t}$ than needed; for example a uniform ellipticity assumption for the generator $\mathcal{L}$ was imposed in [Won66]. This was noticed in the paper [Hai09], where a generalization of the results from [Won66] is stated. Here, we provide the details for this generalization and also phrase the conclusions in a slightly different way. See also Lemma 3.11 of [Kha11].

To formulate the result, for $R>0$ we let

$$
\xi_{R}=\inf \left\{t \geq 0:\left|X_{t}\right| \leq R\right\}
$$


that is, $\xi_{R}$ is the first hitting time of the closed ball of radius $R>0$ centered at the origin in $\mathbb{R}^{n}$. This is a small abuse of notation, see $\xi_{\mathcal{K}}$ in $(2.7)$ above, but there should not be any confusion given the context.

Theorem 2.2. Suppose that there exist $V_{1}, V_{2} \in C^{2}\left(\mathbb{R}^{n} ; \mathbb{R}\right)$ satisfying the following properties:

(p1) $\lim \sup V_{1}(X)=\infty$.

$$
|X| \rightarrow \infty
$$

(p2) $V_{2}$ is strictly positive outside of a compact set.

(p3) $\limsup _{S \rightarrow \infty} \frac{\max _{|X|=S} V_{1}(X)}{\min _{|X|=S} V_{2}(X)}=0$.

(p4) There exists $R>0$ such that

$$
\mathcal{L} V_{1}(X) \geq 0 \quad \text { and } \quad \mathcal{L} V_{2}(X) \leq 1
$$

for every $|X| \geq R$, where $\mathcal{L}$ is the generator for (2.1) given in (2.2).

Then, there exists $M \geq 0$ such that

$$
\mathbb{E}_{X_{*}} \xi_{R}=\infty, \quad \text { whenever }\left|X_{*}\right| \geq R \text { and } V_{1}\left(X_{*}\right) \geq M \text {. }
$$

Proof. First notice that, given $V_{1}, V_{2} \in C^{2}\left(\mathbb{R}^{n} ; \mathbb{R}\right)$ and $R$ satisfying $(p 1)$ - $(p 4)$, one can add a negative constant to $V_{1}$ to obtain

$$
V_{1}(X) \leq 0, \quad \text { for every }|X| \leq R
$$

and a positive constant to $V_{2}$ so that

$$
V_{2}(X) \geq 0 \quad \text { for every } X \in \mathbb{R}^{n} .
$$

Since an addition of constants does not affect (p1) - ( $\left.p_{4}\right)$ we proceed assuming (2.11) and (2.12).

Let us fix an arbitrary $\left|X_{*}\right| \geq R$ such that $V_{1}\left(X_{*}\right)>0$. Invoking (p1) we can choose a sequence of points $x_{k} \in \mathbb{R}^{n}, k=1,2, \cdots$ such that $x_{1}=X_{*}, R<\left|x_{k}\right|<\left|x_{k+1}\right|$ for all $k$ and such that $0<V_{1}\left(x_{k}\right) \uparrow \infty$ as $k \rightarrow \infty$. Let $\tau_{k}^{\prime}=\inf \left\{t \geq 0:\left|X_{t}\right| \geq\left|x_{k}\right|\right\}$ and recalling (2.9) define functions $u_{k}(X, t)$ by

$$
u_{k}(X, t)=\mathbb{E}_{X}\left(\tau_{k}^{\prime} \wedge \xi_{R} \wedge t\right) .
$$

Note that, in particular, since $x_{k}$ is an increasing sequence, we have the relationship

$$
0 \leq u_{k}(X, t) \leq u_{k+1}(X, t)
$$

for all $X$ with $R \leq|X| \leq\left|x_{k}\right|$ and all $k \in \mathbb{N}, t \geq 0$.

Define $M_{k}=\max _{|Y|=\left|x_{k}\right|} V_{1}(Y)$. Note that $M_{1}>0$ and by passing to the relevant subsequence of $x_{n}$ 's via (p1) we can assume that $M_{k+1}>M_{k}$ for all $k$. Let

$$
\lambda_{k}:=M_{k}^{-1} \min _{|Y|=\left|x_{k}\right|} V_{2}(Y)=\frac{\min _{|Y|=\left|x_{k}\right|} V_{2}(Y)}{\max _{|Y|=\left|x_{k}\right|} V_{1}(Y)}
$$

and consider the functions

$$
V(k, X):=\lambda_{k} V_{1}(X)-V_{2}(X)=\frac{\min _{|Y|=\left|x_{k}\right|} V_{2}(Y)}{\max _{|Y|=\left|x_{k}\right|} V_{1}(Y)} V_{1}(X)-V_{2}(X),
$$

for each $k \in \mathbb{N}$. In view of assumption (p3), after passing to a subsequence, and recalling our choice of $X^{*}$ such that $V_{1}\left(X^{*}\right)>0$ we have that

$$
\lim _{k \rightarrow \infty} \lambda_{k}=\infty \text { and } \lim _{k \rightarrow \infty} V\left(k, X^{*}\right)=\infty .
$$

Furthermore, with $(2.11),(2.12)$ we see that $V(k, X)$ is nonpositive on the boundary of the annulus $\mathcal{A}_{k}:=\left\{X: R<|X|<\left|x_{k}\right|\right\}$, namely

$$
V(k, X) \leq 0 \quad \text { for every } X \in\left\{Y \in \mathbb{R}^{n}:|Y|=R \text { or }|Y|=\left|x_{k}\right|\right\},
$$

for every $n$. 
Next, by Dynkin's formula and then invoking (p4) to produces the final inequality, we have

$$
\begin{aligned}
-\mathbb{E}_{X_{*}} V\left(k, X_{\tau_{k}^{\prime} \wedge \xi_{R} \wedge t}\right) & =-V\left(k, X_{*}\right)-\mathbb{E}_{X_{*}} \int_{0}^{\tau_{k}^{\prime} \wedge \xi_{R} \wedge t} \mathcal{L} V\left(k, X_{s}\right) d s \\
& =u_{k}\left(X_{*}, t\right)-V\left(k, X_{*}\right)+\mathbb{E}_{X_{*}} \int_{0}^{\tau_{k}^{\prime} \wedge \xi_{R} \wedge t}\left(\mathcal{L}\left(V_{2}-\lambda_{k} V_{1}\right)\left(X_{s}\right)-1\right) d s \\
& \leq u_{k}\left(X_{*}, t\right)-V\left(k, X_{*}\right) .
\end{aligned}
$$

for any $k \in \mathbb{N}, t \geq 0$. Note that if $\mathbb{E}_{X_{*}}\left(\xi_{R} \wedge \tau_{k}^{\prime}\right)=\infty$ for some $k \in \mathbb{N}$, the desired result follows from the monotone convergence theorem after passing $k \rightarrow \infty$. Thus, we are left with the case $\mathbb{E}_{X_{*}}\left(\xi_{R} \wedge \tau_{k}^{\prime}\right)<\infty$, and in particular $\mathbb{P}\left(\xi_{R} \wedge \tau_{k}^{\prime}<\infty\right)=1$ for all $k$. For fixed $k, V(k, \cdot)$ is bounded and continuous on $\mathcal{A}_{k}$, and therefore by the monotone and dominated convergence theorem after passing $t \rightarrow \infty$ we obtain

$$
-\mathbb{E}_{X_{*}} V\left(k, X_{\tau_{k}^{\prime} \wedge \xi_{R}}\right) \leq \mathbb{E}_{X_{*}}\left(\xi_{R} \wedge \tau_{k}^{\prime}\right)-V\left(k, X_{*}\right) .
$$

Since $\xi_{R} \wedge \tau_{k}^{\prime}<\infty$ is almost surely bounded, (2.14) produces the inequality

$$
V\left(k, X_{*}\right) \leq \mathbb{E}_{X_{*}}\left(\xi_{R} \wedge \tau_{k}^{\prime}\right) \leq \mathbb{E}_{X_{*}} \xi_{R}
$$

valid for all $k \in \mathbb{N}$. Thus invoking (2.13) we conclude (2.10) completing the proof.

In summary Proposition 2.1 and Theorem 2.2 provide a basis for analyzing the expected return time to compact sets for general diffusions of the form (2.1). For our purposes here we can then appeal to general results found in e.g $\left[\mathrm{K}^{+} 87, \mathrm{MT} 12\right]$ to conclude either the existence or the non-existence of an invariant probability measure for $\mathcal{P}_{t}$. Note however that, at this step in the analysis, we further require that $\mathcal{P}_{t}$ maintain certain support and regularity properties.

In order to restate the results from $\left[\mathrm{K}^{+} 87, \mathrm{MT} 12\right]$, we need the following definitions.

Definition 2.3. Suppose that $\mathcal{A}$ is a differential operator defined on an open subset $U \subseteq \mathbb{R}^{n}$. We say that $\mathcal{A}$ is hypoelliptic on $U$ if for any distribution $u$ defined on an open subset $V \subseteq U$ such that $\mathcal{A} u \in C^{\infty}(V)$, we have $u \in C^{\infty}(V)$.

Definition 2.4. We say that $X_{t}$ satisfying (2.1) is nice diffusion if it is non-explosive as in (2.4) and the following conditions are met:

(i) $F \in C^{\infty}\left(\mathbb{R}^{n} ; \mathbb{R}^{n}\right)$ and $G \in C^{\infty}\left(\mathbb{R}^{n} ; M_{n k}\right)$;

(ii) The operators $\mathcal{L}, \mathcal{L}^{*}, \mathcal{L} \pm \partial_{t}, \mathcal{L}^{*} \pm \partial_{t}$ are hypoelliptic on the respective domains $\mathbb{R}^{n}, \mathbb{R}^{n}, \mathbb{R}^{n} \times$ $(0, \infty), \mathbb{R}^{n} \times(0, \infty)$ where $\mathcal{L}^{*}$ denotes the formal adjoint of $\mathcal{L}$ with respect to the $L^{2}\left(\mathbb{R}^{n} ; d x\right)$ inner product.

(iii) $\operatorname{supp}\left(\mathcal{P}_{t}(X, \cdot)\right)=\mathbb{R}^{n}$ for all $t>0, X \in \mathbb{R}^{n} .^{2}$

Note that hypoellipticity of $\mathcal{A}$ on $U$ intuitively means that $\mathcal{A}$ has a local smoothing effect on $U$ reminiscent of elliptic operators. Hypoellipticity of $\mathcal{L}, \mathcal{L}^{*}, \mathcal{L} \pm \partial_{t}, \mathcal{L}^{*} \pm \partial_{t}$ implies their smoothing properties and in addition the probability density functions of the associated stochastic differential equations exist and are smooth in all variables (forward, backward and time). Furthermore if an invariant probability measure exists, the hypoellipticity guarantees the existence and smoothness of an invariant probability density. This is the reason we assume condition (ii) in Definition 2.4.

Proposition 2.5. Suppose that $X_{t}$ is a nice diffusion according to Definition 2.4. Then we have the following:

(a) There is at most one invariant probability measure for $\mathcal{P}_{t}$.

\footnotetext{
${ }^{2}$ Recall that, given a probability measure $\mu$ on $\mathbb{R}^{n}$, $\operatorname{supp}(\mu):=\left\{x \in \mathbb{R}^{n}: \mu(\{y:|y-x|<\epsilon\})>0\right.$, for every $\left.\epsilon>0\right\}$.
}

In particular, $\operatorname{supp}(\mu)=\mathbb{R}^{n}$ if $\mu$ is continuously distributed and its density is almost surely positive. 
(b) $\mathcal{P}_{t}$ has an invariant probability measure if and only if there exists $R>0$ such that $\mathbb{E}_{X} \xi_{R}<\infty$ for all $X \in \mathbb{R}^{n}$ and the mapping $X \mapsto \mathbb{E}_{X} \xi_{R}$ is bounded on compact subsets of $\mathbb{R}^{n}$. In the above, we recall that $\xi_{R}$ is the return time defined in (2.9).

The proof of Proposition 2.5 combines results scattered in the literature; cf. [MT57, Kha60, $\mathrm{K}^{+} 87$, Bel06, Kha11, MT12]. Part (a) is a standard consequence of ergodic decomposition, see, for example [Bel06, Proposition 8.1]. For part (b), if there exists $R>0$ such that $\mathbb{E}_{X} \xi_{R}<\infty$ for all $X \in \mathbb{R}^{n}$ and the mapping $X \mapsto \mathbb{E}_{X} \xi_{R}$ is bounded on compact subsets of $\mathbb{R}^{n}$, the unique invariant probability measure can be constructed using Khasminskii's cycle argument as in [Kha11, Bel06]. The remaining implication in part (b) is more subtle, as it relies on the dichotomy between transient points and recurrent points for degenerate diffusions. This was established in $\left[\mathrm{K}^{+} 87\right]$.

We next recall a set of criteria which can be used to establish the smoothness and positivity hypothesis of Definition 2.4 required for Proposition 2.5. First, we formulate in our setting [HM15b, Theorem 2.9] which is a combination of Hörmander's hypo-ellipticity theorem [Hör67a], ensuring the existence and smoothness of a density (with respect to Lebesgue measure on $\mathbb{R}^{n}$ ), with the support theorems of Stroock and Varadhan [SV72a, SV72b] relating positivity of the density to controllability. By the results from [HM15b], for (1.1), one can use certain Lie brackets as in [Hör67a] to obtain both the regularity of the density and support of the transition measure.

To formulate the result let us introduce preliminary definitions and notations following as closely as possible the formulations in [HM15b]. Recall that the Lie bracket of (smooth) vector fields

$$
U(X)=\sum_{j=1}^{n} U^{j}(X) \frac{\partial}{\partial X_{j}}, \quad W(X)=\sum_{j=1}^{n} W^{j}(X) \frac{\partial}{\partial X_{j}},
$$

is given as

$$
[U, W](X)=\sum_{j=1}^{n} \sum_{k=1}^{n}\left(U^{k}(X) \frac{\partial W^{j}(X)}{\partial X_{k}}-W^{k}(X) \frac{\partial U^{j}(X)}{\partial X_{k}}\right) \frac{\partial}{\partial X_{j}} .
$$

We then introduce, for any vector fields $U$ and $W$

$$
\operatorname{ad}^{0} U(W)=W, \quad \operatorname{ad}^{1} U(W)=[U, W], \quad \operatorname{ad}^{m} U(W):=\operatorname{ad}^{1} U\left(\operatorname{ad}^{m-1} U(W)\right), \text { for } m \geq 2 .
$$

When $W$ is a polynomial vector field (that is to say $W$ depends polynomially on the components of $X)$, for any $X \in \mathbb{R}^{n}$ we denote

$$
\mathfrak{n}(X, W):=\max _{j=1, \ldots, n} \operatorname{deg}\left(p_{j}\right) \quad \text { where } p_{j}(\lambda):=W_{j}(\lambda X) .
$$

For any collection of vector fields $\mathcal{G}$ on $\mathbb{R}^{n}$ we define

$$
\text { cone }_{\geq 0}(\mathcal{G})=\left\{\sum_{j=1}^{N} \lambda_{j} U_{j}: \text { for any finite collections }\left\{\lambda_{1}, \ldots, \lambda_{N}\right\} \subset[0, \infty),\left\{U_{1}, \ldots, U_{N}\right\} \subset \mathcal{G}\right\} .
$$

For simplicity and in the view of (2.1) we restrict to the case when the diffusion coefficients $G$ are independent of $X$ and the drift $F$ is a polynomial. Let

$$
\mathcal{G}_{0}:=\operatorname{span}\left\{G_{1}, \ldots, G_{k}\right\}
$$

and starting at $j=1$ we define ${ }^{3}$

$$
\begin{aligned}
\mathcal{G}_{1}^{O} & :=\mathcal{G}_{0} \cup\left\{\operatorname{ad}^{\mathfrak{n}(G, F)} G(F): G \in \mathcal{G}_{0}, \mathfrak{n}(G, F) \text { is odd }\right\} \\
\overline{\mathcal{G}}_{1}^{O} & :=\left\{G \in \mathcal{G}_{1}^{O}: G \text { is a constant vector field }\right\} \\
\mathcal{G}_{1}^{E} & :=\left\{\operatorname{ad}^{\mathfrak{n}(G, F)} G(F): G \in \mathcal{G}_{0}, \mathfrak{n}(G, F) \text { is even }\right\} \\
\mathcal{G}_{1} & =\operatorname{span}\left(\mathcal{G}_{1}^{O}\right)+\text { cone }_{\geq 0}\left(\mathcal{G}_{1}^{E}\right) .
\end{aligned}
$$

\footnotetext{
${ }^{3}$ Note that in (2.17) and (2.18) we treat constant vector fields $G$ as a vector in $\mathbb{R}^{n}$ when computing $\mathfrak{n}(G, F)$.
} 
We then proceed iteratively to define, for $j \geq 1$

$$
\begin{aligned}
\mathcal{G}_{j+1}^{O} & :=\mathcal{G}_{j}^{O} \cup\left\{\operatorname{ad}^{\mathfrak{n}(G, F)} G(H): G \in \overline{\mathcal{G}}_{j}^{O}, H \in \mathcal{G}_{j}, \mathfrak{n}(G, H) \text { is odd }\right\} \\
\overline{\mathcal{G}}_{j+1}^{O} & :=\left\{G \in \mathcal{G}_{j+1}^{O}: G \text { is a constant vector field }\right\} \\
\mathcal{G}_{j+1}^{E} & :=\mathcal{G}_{j}^{E} \cup\left\{\operatorname{ad}^{\mathfrak{n}(G, F)} G(H): G \in \overline{\mathcal{G}}_{j}^{O}, H \in \mathcal{G}_{j}, \mathfrak{n}(G, H) \text { is even }\right\} \\
\mathcal{G}_{j+1} & :=\operatorname{span}\left(\mathcal{G}_{j+1}^{O}\right)+\text { cone }_{\geq 0}\left(\mathcal{G}_{j+1}^{E}\right) .
\end{aligned}
$$

The following summarizes results in [HM15b]; cf. [Hör67a, SV72a, SV72b, Bel06].

Theorem 2.6. Consider $\left\{X_{t}\right\}_{t \geq 0}$ solving (2.1) under the assumption that $F$ is a polynomial, that $G_{k}$ is constant i.e. $X$-independent and suppose furthermore that the resulting dynamics is non-explosive as in (2.4). Assume that

$$
\operatorname{span}\left\{H \in \bigcup_{j \geq 1} \mathcal{G}_{j}^{O}: H \text { is a constant vector field }\right\}=\mathbb{R}^{n},
$$

then $\left\{X_{t}\right\}$ is a nice diffusion in the sense of Definition 2.4.

Remark 2.7. The condition (2.19) is special case of the Hörmander (parabolic) sum of squares condition which asserts that if vector fields produced by the iterated Lie brackets

$$
G_{1}, \ldots, G_{k},\left[G_{1}, F\right], \ldots,\left[G_{k}, F\right],\left[\left[G_{1}, F\right], F\right],\left[\left[G_{1}, F\right], G_{1}\right] \ldots
$$

span all of $\mathbb{R}^{n}$ then the generator $\mathcal{L}$ given by (2.2) along with $\mathcal{L}^{*}, \mathcal{L} \pm \partial_{t}, \mathcal{L}^{*} \pm \partial_{t}$ are all hypo-elliptic as in Definition 2.3. See [Hör67b] and more recently the treatment in [Bel06].

\section{Non-explosivity and Uniqueness Results}

In this section we now return to the specific setting (1.1) and establish, subject to a non-degeneracy condition on the noise, the hypo-ellipticity and irreducibility of (1.1). Specifically we establish that (1.1) satisfies Definition 2.4 via Theorem 2.6 when $\gamma_{1}>0$ and at least one of $\gamma_{2}, \gamma_{3}$ is strictly positive.

Let $\left\{X_{t}\right\}_{t \geq 0}$ denote the process $\left(x_{t}, y_{t}, z_{t}\right)$ solving (1.1), and we will reuse the notations $\tau_{n}, \tau, \mathcal{L}, \mathcal{P}_{t}$, etc. from Section 2 for $\left\{X_{t}\right\}_{t \geq 0}$. In particular note that (1.1) produces the infinitesimal generator

$$
\mathcal{L}=\sigma(y-x) \partial_{x}+[x(\rho-z)-y] \partial_{y}+[x y-\beta z] \partial_{z}+\gamma_{1} \partial_{x}^{2}+\gamma_{2} \partial_{y}^{2}+\gamma_{3} \partial_{z}^{2} .
$$

We now formulate the first result in this section.

Proposition 3.1. For any values $\sigma, \rho, \beta \in \mathbb{R}$ and any $\gamma_{1}, \gamma_{2}, \gamma_{2} \geq 0$ the process $\left\{X_{t}\right\}_{t \geq 0}$ defined by (1.1) is nonexplosive in the sense of (2.4). Moreover, if $\sigma>0$ and either $\gamma_{1}, \gamma_{2}>0$ or $\gamma_{1}, \gamma_{3}>0$, then (1.1) is a nice diffusion in the sense of Definition 2.4. Hence, in particular, the hypotheses of Proposition 2.5 are satisfied for (1.1) if $\gamma_{1}, \gamma_{2}>0$ or $\gamma_{1}, \gamma_{3}>0$.

Proof. We first prove that $\left\{X_{t}\right\}_{t \geq 0}$ is non-explosive with the aid of Proposition 2.1. Defining $H$ as in (1.4) we find that (1.5) holds. Thus taking $V=H$ we obtain (2.5) from (1.5) with Young's inequality, and the first assertion follows.

To prove that $\left\{X_{t}\right\}_{t \geq 0}$ is a nice diffusion we proceed via Theorem 2.6. Adopting the geometric notations as in (2.15) we have

$$
F=\sigma(y-x) \partial_{x}+[x(\rho-z)-y] \partial_{y}+[x y-\beta z] \partial_{z}, \quad G_{1}=\sqrt{2 \gamma_{1}} \partial_{x}, \quad G_{2}=\sqrt{2 \gamma_{2}} \partial_{y}, \quad G_{3}=\sqrt{2 \gamma_{3}} \partial_{y} .
$$

Our task is now to exhibit a sequence of allowable Lie brackets between these fields to obtain the spanning condition (2.19).

Start with the case $\gamma_{1}, \gamma_{2}>0$ and by viewing $G_{1}$ as the vector $\left(\sqrt{2 \gamma_{1}}, 0,0\right)^{T}$, we have $F(\lambda G)=$ $\left(-\lambda \sigma \sqrt{2 \gamma_{1}}, \lambda \rho \sqrt{2 \gamma_{1}}, 0\right)^{T}$, and therefore, cf. (2.16), $\mathfrak{n}\left(G_{1}, F\right)=1$. Hence, by (2.17), we find that

$$
G_{1}^{\prime}:=\operatorname{ad}^{1} G_{1}(F)=\left[G_{1}, F\right]=-\sqrt{2 \gamma_{1}} \sigma \partial_{x}+\sqrt{2 \gamma_{1}}(\rho-z) \partial_{y}+\sqrt{2 \gamma_{1}} y \partial_{z} \in \mathcal{G}_{1}^{O}
$$


Next, from $\mathfrak{n}\left(G_{2}, G_{1}^{\prime}\right)=1$ and (2.18) follows

$$
\tilde{G}_{3}:=\operatorname{ad}^{1} G_{2}\left(G_{1}^{\prime}\right)=\left[G_{2}, G_{1}^{\prime}\right]=\sqrt{2 \gamma_{1} \gamma_{2}} \partial_{z} \in \mathcal{G}_{2}^{O} .
$$

Thus we have found $G_{1}, G_{2}, \tilde{G}_{3} \in \bigcup_{j \geq 1} \mathcal{G}_{j}^{O}$ which together span $\mathbb{R}^{3}$ and hence satisfy (2.19), completing the proof in the first case.

Next, assume $\gamma_{1}, \gamma_{3}>0$. As above again $\mathfrak{n}\left(G_{1}, F\right)=1$ and (3.2) holds true. On the other hand, $\mathfrak{n}\left(G_{3}, G_{1}^{\prime}\right)=1$ and we compute

$$
\tilde{G}_{2}:=\operatorname{ad}^{1} G_{3}\left(G_{1}^{\prime}\right)=\left[G_{3}, G_{1}^{\prime}\right]=-\sqrt{2 \gamma_{1} \gamma_{3}} \partial_{y} \in \mathcal{G}_{2}^{O} .
$$

Here, we found the spanning set $G_{1}, \tilde{G}_{2}, G_{3} \in \bigcup_{j \geq 1} \mathcal{G}_{j}^{O}$ satisfying (2.19) as required by Theorem 2.6. The proof is now complete.

\section{Positive Recurrence in the Absence of Damping}

In this section, we study the dynamics (1.1) in the case when $\beta=0$ and $\gamma_{1}>0$. Our goal is to show that (1.1) has globally finite expected returns to some compact set by constructing a Lyapunov function $V$ satisfying the condition (2.6) in Proposition 2.1 part (b). In turn, this result immediately implies the existence part of Theorem 1.2 (ii) as well as the uniqueness in the case when either $\gamma_{1}, \gamma_{2}>0$ or $\gamma_{1}, \gamma_{3}>0$ by way of Proposition 3.1.

We state the main result of this section precisely as follows:

Proposition 4.1. Consider (1.1) in the case when $\sigma>0, \beta=0, \rho \in \mathbb{R}, \gamma_{1}>0$ and $\gamma_{2}, \gamma_{3} \geq 0$. Then, there exists an $R>0$ such that for any $S>0$

$$
\sup _{|X| \leq S} \mathbb{E}_{X} \xi_{R}<\infty
$$

where $\xi_{R}$ is return time to the ball of radius $R$ as defined in (2.9). Furthermore, when we make the additional assumption that either $\gamma_{2}>0$ or $\gamma_{3}>0$ then (1.1) has a unique invariant probability measure.

Regarding the organization of the section, Section 4.1, 4.2, 4.3 contain the derivation of a Lyapunov function $V: \mathbb{R}^{3} \rightarrow \mathbb{R}$ leading to (4.1) and the quantitative estimates implying (2.6). The rigorous proof of Proposition 4.1 is given in Section 4.4.

4.1. Derivation of the Lyapunov Function. In order to simplify our analysis slightly in what follows we begin with the preliminary observation that it is sufficient to address special case when $\rho=0$ in (1.1), namely

$$
\begin{aligned}
& d x=\sigma(y-x) d t+\sqrt{2 \gamma_{1}} d B_{1}, \\
& d y=-x z d t-y d t+\sqrt{2 \gamma_{2}} d B_{2}, \\
& d z=x y d t+\sqrt{2 \gamma_{3}} d B_{3} .
\end{aligned}
$$

Indeed, in the rest of this section we proceed to construct a function $V \in C^{2}\left(\mathbb{R}^{3} ;[0, \infty)\right)$ such that for some constants $c, d>0$ and some compact set $\mathcal{K} \subseteq \mathbb{R}^{3}$ we have

$$
\mathcal{M} V \leq-c+d \mathbf{1}_{\mathcal{K}}
$$

where $\mathcal{M}$ is the infinitesimal generator of (4.2), that is,

$$
\mathcal{M}=\sigma(y-x) \partial_{x}-(x z+y) \partial_{y}+x y \partial_{z}+\gamma_{1} \partial_{x}^{2}+\gamma_{2} \partial_{y}^{2}+\gamma_{3} \partial_{z}^{2} .
$$

Having found such $V$ we obtain according to Proposition 2.1,(b) that

$$
\mathbb{E}_{X} \tilde{\xi}_{\mathcal{K}} \leq \frac{V(X)}{c}, \quad \text { where } \tilde{\xi}_{\mathcal{K}}:=\inf \left\{t \geq 0: \tilde{X}_{t} \in \mathcal{K}\right\}
$$


where $\tilde{X}_{t}=\left(\tilde{x}_{t}, \tilde{y}_{t}, \tilde{z}_{t}\right)$ obeys (4.2). Clearly $X_{t}=\left(\tilde{x}_{t}, \tilde{y}_{t}, \tilde{z}_{t}-\rho\right)$ satisfies (1.1) in the general case of any $\rho \in \mathbb{R}$. Thus, if for any $R>\rho$ we denote

$$
\xi_{R}:=\inf \left\{t \geq 0:\left|X_{t}\right| \leq R\right\}, \quad \tilde{\xi}_{R}:=\inf \left\{t \geq 0:\left|\tilde{X}_{t}\right| \leq R-\rho\right\}
$$

then we have $\xi_{R} \leq \tilde{\xi}_{R}$. Thus, by choosing $R>0$ sufficiently large so that $\mathcal{K} \subset B_{R-\rho}$ we obtain that $\xi_{R} \leq \tilde{\xi}_{R} \leq \tilde{\xi}_{\mathcal{K}}$, so that

$$
\mathbb{E}_{X} \xi_{R} \leq \mathbb{E}_{X} \tilde{\xi}_{R} \leq \frac{V(X)}{c}
$$

allowing us to conclude (4.1) as desired in Proposition 4.1.

In order to find $V$ satisfying (4.3), we first use the natural Lyapunov function for (4.2) when $\beta>0$. Indeed, defining

$$
\tilde{H}(x, y, z)=x^{2}+y^{2}+z^{2}-2 \sigma z+\kappa_{0}
$$

where $\kappa_{0}>0$ is large enough so that $\tilde{H} \geq 0$. Observe that $\tilde{H}$ provides a good initial guess for $V$ since

$$
\mathcal{M}(\tilde{H})=-2 \sigma x^{2}-2 y^{2}+2\left(\gamma_{1}+\gamma_{2}+\gamma_{3}\right),
$$

and therefore we have the desired inequality (4.3) on the set where $|(x, y)|:=\sqrt{x^{2}+y^{2}}$ is large. More specifically, for the region

$$
\mathcal{R}_{0}=\left\{x^{2}+y^{2} \geq R_{0}\right\}
$$

with a sufficiently large $R_{0} \geq 1$ depending only on $\gamma_{1}+\gamma_{2}+\gamma_{3}>0$, we have

$$
\mathcal{M}(\tilde{H}) \leq-\left(\gamma_{1}+\gamma_{2}+\gamma_{3}\right) \quad \text { in } \mathcal{R}_{0} .
$$

However, (4.6) does not imply the bound (4.3) if $|(x, y)|$ is small (and $|z|$ is large).

To fix this issue, we seek for a lower-order perturbation $\psi$ of $\tilde{H}$ encapsulating the 'averaging' effects of the dynamics. More specifically, we start with $\tilde{H}$ and find a function $\psi \in C^{2}\left(\mathbb{R}^{3} ; \mathbb{R}\right)$ satisfying

$$
\limsup _{|X| \rightarrow \infty} \frac{\psi(X)}{\tilde{H}(X)}=0
$$

so that $V:=\tilde{H}+\psi$ satisfies $V \geq 0$ and (4.3) for some $c, d>0$ and the compact set

$$
\mathcal{K}:=\left\{x^{2}+y^{2} \leq R_{0},|z| \leq R_{3}\right\}
$$

for suitable choices of $R_{0}, R_{3} \geq 1$.

Note that, with this strategy, because $\tilde{H}$ satisfies (4.5) on $\mathcal{R}_{0}$, we should naturally set $\psi=0$ on $\mathcal{R}_{0}$. On the other hand, when $x^{2}+y^{2} \leq R_{0}$ and $|z|$ is large, we should seek a nontrivial perturbation $\psi$ through a scaling analysis to identify dominant terms in $\mathcal{M}$.

4.2. Scaling Arguments and Definition of $\psi$. To see how to define $\psi$ on the complement of $\mathcal{R}_{0}$, it is helpful to first heuristically analyze the dynamics when $|z|$ is large and $x$ and $y$ are bounded. To this end, consider the scaling transformation

$$
T_{\lambda}(x, y, z)=\left(\lambda^{-\alpha} x, y, \lambda z\right)
$$

where $\lambda>1$ is large and $\alpha \in[0,1]$. We apply $T_{\lambda}$ to the generator $\mathcal{M}$ to formally see how the dynamics behaves as $z$ gets large. Observe that

$$
\begin{aligned}
T_{\lambda} \circ \mathcal{M} & =\sigma\left(y \lambda^{\alpha}-x\right) \partial_{x}-\left(\lambda^{1-\alpha} x z+y\right) \partial_{y}+\lambda^{-1-\alpha} x y \partial_{z}+\gamma_{1} \lambda^{2 \alpha} \partial_{x}^{2}+\gamma_{2} \partial_{y}^{2}+\lambda^{-2} \gamma_{3} \partial_{z}^{2} \\
& \sim \lambda^{1-\alpha} x z \partial_{y}+\gamma_{1} \lambda^{2 \alpha} \partial_{x}^{2},
\end{aligned}
$$

whenever $\lambda \gg 1$ and $\alpha>0$.

Observe that there are two regimes depending on $\alpha$. If $\alpha \in[0,1 / 3)$, the most significant term in (4.9) is $\lambda^{1-\alpha} x z \partial_{y}$. Hence, the dominant dynamics of (4.2) is given by

$$
\dot{X}=0, \quad \dot{Y}=-X Z, \quad \dot{Z}=0
$$


and we expect such an approximation to be valid in the region

$$
\mathcal{R}_{1}:=\left\{x^{2}+y^{2} \leq R_{0},|x||z|^{1 / 3} \geq R_{1},|z| \geq R_{3}\right\}
$$

where $R_{0}, R_{1}, R_{3} \geq 1$ are large constants to be determined below. This suggests that we search for a function $\psi=\psi_{1}$ such that the infinitesimal generator of (4.10) applied to $\psi_{1}$ is negative

$$
-x z \partial_{y} \psi_{1}=-\kappa_{1}
$$

where $\kappa_{1}>2\left(\gamma_{1}+\gamma_{2}+\gamma_{3}\right)$ is a constant. Note that this equation gives the following particular solution

$$
\psi_{1}=\kappa_{1} \frac{y}{x z} .
$$

In addition, on the set $\mathcal{R}_{1}$ and positivity condition (4.7) holds and ( $\mathcal{M}$ is the generator of (4.2))

$$
\begin{aligned}
\frac{\mathcal{M}\left(\psi_{1}\right)}{\kappa_{1}} & =-\frac{\sigma(y-x) y}{x^{2} z}-1-\frac{y}{z x}-\frac{y^{2}}{z^{2}}+2 \gamma_{1} \frac{y}{x^{3} z}+2 \gamma_{3} \frac{y}{x z^{3}} \\
& \leq-1+C \frac{R_{0}^{3}}{R_{1}}
\end{aligned}
$$

where we used that on $\mathcal{R}_{1}$ one has $|z|^{\frac{1}{3}} \geq \frac{R_{1}}{R_{0}}$ and $R_{i} \geq 1$ for each $i=1,2,3$. Note that the constant $C=C\left(\sigma, \gamma_{3}\right)>0$ is independent of $R_{0}, R_{1}, R_{2}$, and $\kappa_{1}$. Thus, for sufficiently large $R_{1}$ depending on $R_{0}$, we obtain

$$
\mathcal{M}\left(\psi_{1}\right) \leq-\frac{1}{2} \kappa_{1} \quad \text { in } \mathcal{R}_{1}
$$

Consequently, for any fixed $R_{0} \geq 1$, we can choose suitably large $\kappa_{1} \geq 1 \vee\left(4\left(\gamma_{1}+\gamma_{2}+\gamma_{3}\right)\right.$ and $R_{1} \geq 1$ so that

$$
\mathcal{M}\left(\tilde{H}+\psi_{1}\right) \leq-\frac{\kappa_{1}}{2} \quad \text { on the region } \mathcal{R}_{1} .
$$

Next, assume $\alpha \in(1 / 3, \infty)$ and observe that the dominant term in $(4.9)$ is $\gamma_{1} \lambda^{2 \alpha} \partial_{x}^{2}$. See Remark 4.2 below which discusses the boundary case $\alpha=1 / 3$, where the two terms in (4.9) balance. Therefore, the main contribution of the dynamics of (1.1) in the region

$$
\mathcal{R}_{2}=\left\{x^{2}+y^{2} \leq R_{2},|x||z|^{1 / 3} \leq R_{1},|z| \geq R_{3}\right\}
$$

is given by the SDE

$$
d X=\sqrt{2 \gamma_{1}} d B_{1}, \quad \dot{Y}=0, \quad \dot{Z}=0 .
$$

In the definition of $\mathcal{R}_{2}$, the constants $R_{2}$ and $R_{3}$ are considered sufficiently large, possibly depending on $R_{0}$. ${ }^{4}$ Thus, as above, in $\mathcal{R}_{2}$, we should look for $\psi=\psi_{2}$ for which the infinitesimal generator of (4.13) is negative, that is, for $\psi_{2}$ that solves

$$
\gamma_{1} \partial_{x}^{2} \psi_{2}=-\kappa_{2}
$$

where again $\kappa_{2} \geq 1 \vee\left(4\left(\gamma_{1}+\gamma_{2}+\gamma_{3}\right)\right.$ is a large free parameter we can adjust as suits our needs further on. Note that a particular solution of this partial differential equation is

$$
\psi_{2}=\frac{\kappa_{2}}{2 \gamma_{1}}\left(\frac{4 R_{1}^{2}}{|z|^{2 / 3}}-x^{2}\right) .
$$

The solution is chosen such that it satisfies

$$
\left|\psi_{2}\right| \leq C \frac{\kappa_{2} R_{1}^{2}}{|z|^{2 / 3}} \quad \text { whenever }|x||z|^{1 / 3} \leq 2 R_{1} .
$$

\footnotetext{
${ }^{4}$ Note that additional parameter $R_{2}$ can be simply taken as $R_{0}$ in our preliminary analysis. However, it will play an important role later when we need to 'glue' our Lypunov function $V$ together to obtain a $C^{2}$ function.
} 
As for the previous case, one can easily check (4.7), that is, $\psi_{2}$ is dominated by $\tilde{H}$ for large values of $(x, y, z) \in \mathcal{R}_{2}$. Moreover $\psi_{2}$ satisfies, for $z \neq 0$,

$$
\mathcal{M}\left(\psi_{2}\right)=-\kappa_{2}-\frac{\kappa_{2} \sigma}{\gamma_{1}} x(y-x)-\frac{R_{1}^{2} \kappa_{2}}{3 \gamma_{1}} \frac{x y}{|z|^{2 / 3}}+\frac{10 \kappa_{2} R_{1}^{2}}{9} \frac{\gamma_{3}}{\gamma_{1}} \frac{1}{|z|^{8 / 3}} .
$$

Also, in $\mathcal{R}_{2}$, using that $|x| \leq R_{1} /|z|^{1 / 3}$ and $R_{i} \geq 1$ for $i=1,2,3$, we have

$$
\frac{\kappa_{2} \sigma}{\gamma_{1}}|x(y-x)| \leq \frac{2 \kappa_{2} \sigma}{\gamma_{1}} \frac{R_{1} R_{2}^{1 / 2}}{|z|^{1 / 3}} \leq \frac{2 \kappa_{2} \sigma}{\gamma_{1}} \frac{R_{1}^{2} R_{2}}{R_{3}^{1 / 3}} .
$$

Since the other terms (except $-\kappa_{2}$ ) have $|z|$ to some power in the denominator, they are straightforward to estimate. Overall, it follows

$$
\mathcal{M}\left(\psi_{2}\right) \leq-\kappa_{2}\left(1-\frac{C R_{1}^{2} R_{2}}{R_{3}^{1 / 3}}\right),
$$

where the constant $C=C\left(\sigma, \gamma_{1}, \gamma_{3}\right)$ is independent of $R_{1}, R_{3}, R_{3}$, and $\kappa_{2}$. Hence, given $R_{2}, R_{1} \geq 1$, we choose large $R_{3} \geq 1$ and $\kappa_{2} \geq 1 \vee\left(4\left(\gamma_{1}+\gamma_{2}+\gamma_{3}\right)\right.$ so that

$$
\mathcal{M}\left(\tilde{H}+\psi_{2}\right) \leq-\frac{\kappa_{2}}{2} \quad \text { in } \mathcal{R}_{2}
$$

Let us now make the preliminary definition

$$
V:=\tilde{H}+\mathbb{1}_{\mathcal{R}_{1}} \psi_{1}+\mathbb{1}_{\mathcal{R}_{2}} \psi_{2}
$$

and notice that the complement of compact region $\mathcal{K}=\left\{x^{2}+y^{2} \leq R_{0}, z \leq R_{1}\right\}$, as in (4.8) satisfies

$$
\mathcal{K}^{C} \subseteq \mathcal{R}_{0} \cup \mathcal{R}_{1} \cup \mathcal{R}_{2}
$$

Thus, setting aside the issue of differentiability of $V$, we can choose values for $R_{0}, R_{1}, R_{2}, R_{3} \geq 1$ and values for $\kappa_{1}, \kappa_{2} \geq 4\left(\gamma_{1}+\gamma_{2}+\gamma_{3}\right)$ such that a combination of (4.6), (4.12) and (4.16) leads to (4.3).

The next section addresses the smoothness issue for $V$ defined as (4.17) by replacing indicator functions with smooth cut-off functions. We also provide estimates for additional terms which are produced when the operator $\mathcal{M}$ acts on these smooth cut-offs.

Remark 4.2. One may be concerned that, when defining $\psi_{1}$ and $\psi_{2}$ we neglected the effective dynamics of (1.1) in the critical region $\alpha=1 / 3$. This is not a problem because the function $\psi_{2}$ is independent of $y$, and therefore it solves the associated PDE with both dominant terms

$$
-x z \partial_{y} \psi_{2}+\gamma_{1} \partial_{x}^{2} \psi_{2}=-\kappa_{2} .
$$

4.3. Gluing. In order to replace the indicator functions in (4.17) with smooth cut-off functions we adopt the following definitions. Consider $\chi$ and $\tilde{\chi}$ to be non-negative $C^{\infty}(\mathbb{R})$ functions such that

$$
\chi(x)=\left\{\begin{array}{ll}
1 & \text { if }|x| \leq 1 \\
0 & \text { if }|x| \geq 2
\end{array} \quad \text { and } \quad \tilde{\chi}(x)= \begin{cases}1 & \text { if }|x| \geq 1 \\
0 & \text { if }|x| \leq 1 / 2 .\end{cases}\right.
$$

We now define ${ }^{5}$

$$
\theta_{1}(x, y, z):=\chi\left(\frac{x^{2}+y^{2}}{R_{0}}\right) \tilde{\chi}\left(\frac{|x||z|^{1 / 3}}{R_{1}}\right) \tilde{\chi}\left(\frac{|z|}{R_{3}}\right):=\theta_{1}^{0}(x, y) \tilde{\theta}_{1}^{1}(x, z) \tilde{\theta}^{3}(z)
$$

and put

$$
\theta_{2}(x, y, z):=\chi\left(\frac{x^{2}+y^{2}}{R_{2}}\right) \chi\left(\frac{|x||z|^{1 / 3}}{R_{1}}\right) \tilde{\chi}\left(\frac{|z|}{R_{3}}\right):=\theta_{2}^{2}(x, y) \theta_{2}^{1}(x, z) \tilde{\theta}^{3}(z)
$$

\footnotetext{
${ }^{5}$ Observe that for example $\tilde{\theta}_{2}^{3}(z)$ indicates that we are cutting off the region in $z$ (argument of the function) below the parameter value $R_{3}$ (tilde and superscript).
} 
We now define

$$
\begin{aligned}
V & :=\tilde{H}+\theta_{1} \psi_{1}+\theta_{2} \psi_{2} \\
& =x^{2}+y^{2}+z^{2}-2 \sigma z+\kappa_{0}+\kappa_{1} \theta_{1}(x, y, z) \frac{y}{x z}+\kappa_{2} \theta_{2}(x, y, z) \frac{1}{2 \gamma_{1}}\left(\frac{R_{1}^{2}}{|z|^{2 / 3}}-x^{2}\right) .
\end{aligned}
$$

Of course this definition requires the specification of the parameters $R_{0}, R_{1}, R_{2}, R_{3} \geq 1$ and $\kappa_{0}, \kappa_{1}, \kappa_{2}>$ 0 , which will be clarified as we proceed with the argument.

4.4. Rigorous Bounds on $V$. We are now ready to use $V$ defined in (4.20) to prove the main result of this section.

Proof of Proposition 4.1. As we identified in the argumentation leading to (4.4) above, it is sufficient to show that the $V$ defined by (4.20) satisfies (4.3) and is strictly positive for suitable values of $R_{0}, R_{1}, R_{2}, R_{3}$ and $\kappa_{0}, \kappa_{1}, \kappa_{2}$. We emphasize that for the remainder of the proof, any constant $C>0$ is independent of the values of the parameters $R_{0}, R_{1}, R_{2}, R_{3}$ and $\kappa_{0}, \kappa_{1}, \kappa_{2}$ unless explicitly stated otherwise.

Regarding the condition (4.3) we begin by observing that

$$
\begin{aligned}
\mathcal{M}(V)= & \mathcal{M}(\tilde{H})+\theta_{1} \mathcal{M}\left(\psi_{1}\right)+\theta_{2} \mathcal{M}\left(\psi_{2}\right) \\
& +\psi_{1} \mathcal{M}\left(\theta_{1}\right)+2 \nabla_{\gamma} \theta_{1} \cdot \nabla_{\gamma} \psi_{1}+\psi_{2} \mathcal{M}\left(\theta_{2}\right)+2 \nabla_{\gamma} \theta_{2} \cdot \nabla_{\gamma} \psi_{2},
\end{aligned}
$$

where we adopt the shorthand notation $\nabla_{\gamma}=\left(\gamma_{1} \partial_{x}, \gamma_{2} \partial_{y}, \gamma_{3} \partial_{z}\right)$. We proceed to expand each of the terms in (4.21), where derivatives fall on the cut-off functions $\theta_{1}$ and $\theta_{2}$. For later use we note the estimate

$$
\left|\partial_{s}^{i} \theta_{1}^{0}\right| \leq C \mathbb{1}_{x^{2}+y^{2} \leq 2 R_{0}}
$$

where $s$ stands for $x$ or $y$ and $i \in\{1,2\}$. Indeed, for example

$$
\left|\partial_{y}^{2} \theta_{1}^{0}\right| \leq C\left(\frac{1}{R_{0}}+\frac{|y|^{2}}{R_{0}^{2}}\right) \mathbb{1}_{x^{2}+y^{2} \leq 2 R_{0}} \leq C \mathbb{1}_{x^{2}+y^{2} \leq 2 R_{0}}
$$

and other estimates follow analogously. In addition, we have

$$
\begin{gathered}
\left|\partial_{x} \tilde{\theta}_{1}^{1}\right| \leq C \frac{|z|^{1 / 3}}{R_{1}}, \quad\left|\partial_{x}^{2} \tilde{\theta}_{1}^{1}\right| \leq C \frac{|z|^{2 / 3}}{R_{1}^{2}}, \quad\left|\partial_{z} \tilde{\theta}_{1}^{1}\right| \leq C \frac{|x|}{|z|^{2 / 3} R_{1}}, \quad\left|\partial_{z}^{2} \tilde{\theta}_{1}^{1}\right| \leq C\left(\frac{|x|}{|z|^{5 / 3} R_{1}}+\frac{|x|^{2}}{|z|^{4 / 3} R_{1}^{2}}\right), \\
\left|\partial_{z} \tilde{\theta}^{3}\right| \leq C \frac{1}{R_{3}} \mathbb{1}_{|z| \geq R_{3} / 2}, \quad\left|\partial_{z}^{2} \tilde{\theta}^{3}\right| \leq C \frac{1}{R_{3}^{2}} \mathbb{1}_{|z| \geq R_{3} / 2}
\end{gathered}
$$

for a constant $C$ depending only and on the specifics of the cut-offs $\chi$ and $\tilde{\chi}$ independent of $R_{0}, R_{1}$, and $R_{3}$. Similarly

$$
\left|\partial_{s}^{i} \theta_{2}^{2}\right| \leq C \mathbb{1}_{x^{2}+y^{2} \leq 2 R_{2}}
$$

where $s$ stands for $x$ or $y$ and $i \in\{1,2\}$ and

$$
\left|\partial_{x} \theta_{2}^{1}\right| \leq C \frac{|z|^{1 / 3}}{R_{1}}, \quad\left|\partial_{x}^{2} \theta_{2}^{1}\right| \leq C \frac{|z|^{2 / 3}}{R_{1}^{2}}, \quad\left|\partial_{z} \theta_{2}^{1}\right| \leq C \frac{|x|}{|z|^{2 / 3} R_{1}}, \quad\left|\partial_{z}^{2} \theta_{2}^{1}\right| \leq C\left(\frac{|x|}{|z|^{5 / 3} R_{1}}+\frac{|x|^{2}}{|z|^{4 / 3} R_{1}^{2}}\right),
$$

where again $C>0$ is independent of $R_{1}, R_{2}$, and $R_{3}$. Observe that $\tilde{\theta}^{3}$ is the same in both $\theta_{1}$ and $\theta_{2}$. Denote $K_{R_{3}}$ a constant that might depend on $R_{0}, R_{1}$, and $R_{2}$ such that

$$
\lim _{R_{3} \rightarrow \infty} K_{R_{3}}=0 \text {. }
$$


We expand $\psi_{1} \mathcal{M}\left(\theta_{1}\right)$ as

$$
\begin{aligned}
\psi_{1} \mathcal{M}\left(\theta_{1}\right)= & \sigma(y-x) \psi_{1}\left(\partial_{x} \theta_{1}^{0} \tilde{\theta}_{1}^{1} \tilde{\theta}^{3}+\theta_{1}^{0} \partial_{x} \tilde{\theta}_{1}^{1} \tilde{\theta}^{3}\right)-(x z+y) \psi_{1} \partial_{y} \theta_{1}^{0} \tilde{\theta}_{1}^{1} \tilde{\theta}^{3}+x y \psi_{1}\left(\theta_{1}^{0} \partial_{z} \tilde{\theta}_{1}^{1} \tilde{\theta}^{3}+\theta_{1}^{0} \tilde{\theta}_{1}^{1} \partial_{z} \tilde{\theta}^{3}\right) \\
& +\gamma_{1} \psi_{1}\left(\partial_{x}^{2} \theta_{1}^{0} \tilde{\theta}_{1}^{1} \tilde{\theta}^{3}+\theta_{1}^{0} \partial_{x}^{2} \tilde{\theta}_{1}^{1} \tilde{\theta}^{3}+2 \partial_{x} \theta_{1}^{0} \partial_{x} \tilde{\theta}_{1}^{1} \tilde{\theta}^{3}\right)+\gamma_{2} \psi_{1} \partial_{y}^{2} \theta_{1}^{0} \tilde{\theta}_{1}^{1} \tilde{\theta}^{3} \\
& +\gamma_{3} \psi_{1}\left(\theta_{1}^{0} \partial_{z}^{2} \tilde{\theta}_{1}^{1} \tilde{\theta}^{3}+\theta_{1}^{0} \tilde{\theta}_{1}^{1} \partial_{z}^{2} \tilde{\theta}^{3}+2 \theta_{1}^{0} \partial_{z} \tilde{\theta}_{1}^{1} \partial_{z} \tilde{\theta}^{3}\right) .
\end{aligned}
$$

Since on $\mathcal{R}_{1}$ one has

$$
\left|\psi_{1}\right|=\kappa_{1}\left|\frac{y}{x z}\right| \leq \frac{\kappa_{1} R_{0}^{\frac{1}{2}}}{R_{1}} \frac{1}{|z|^{\frac{2}{3}}}
$$

and $x, y$ are bounded, it is easy to check that all terms except $x z \psi_{1} \partial_{y} \theta_{1}^{0} \tilde{\theta}_{1}^{1} \tilde{\theta}_{1}^{3}$ and $\gamma_{1} \psi_{1} \theta_{1}^{0} \partial_{x}^{2} \tilde{\theta}_{1}^{1} \tilde{\theta}_{1}^{3}$ can be bounded by $\kappa_{1} K_{R_{3}}$ (some power of $z$ is left in the denominator). Referring back to (4.22), (4.23), and (4.26) we have

$$
\begin{aligned}
& \left|x z \psi_{1} \partial_{y} \theta_{1}^{0} \tilde{\theta}_{1}^{1} \tilde{\theta}^{3}\right|=\kappa_{1}\left|y \partial_{y} \theta_{1}^{0} \tilde{\theta}_{1}^{1} \tilde{\theta}^{3}\right| \leq C \kappa_{1} \mathbb{1}_{R_{0} \leq x^{2}+y^{2} \leq 2 R_{0}} \leq C \kappa_{1} \mathbb{1}_{\mathcal{R}_{0}} \\
& \left|\gamma_{1} \psi_{1} \theta_{1}^{0} \partial_{x}^{2} \tilde{\theta}_{1}^{1} \tilde{\theta}^{3}\right| \leq C \frac{\kappa_{1} R_{0}^{\frac{1}{2}}}{R_{1}} \frac{1}{|z|^{\frac{2}{3}}} \frac{|z|^{\frac{2}{3}}}{R_{0}^{2}} \leq C \kappa_{1} \frac{1}{R_{1}}
\end{aligned}
$$

where the constant $C$ is independent of $R_{0}, R_{1}, R_{2}, R_{3}$ and $\kappa_{0}, \kappa_{1}, \kappa_{2}$. Overall, we have

$$
\left|\psi_{1} \mathcal{M}\left(\theta_{1}\right)\right| \leq C \kappa_{1}\left(\mathbb{1}_{\mathcal{R}_{0}}+\frac{1}{R_{1}}+K_{R_{3}}\right)
$$

where we recall that $K_{R_{3}}$ is as in (4.24).

Next, we estimate

$$
\begin{aligned}
& \left|\nabla_{\gamma} \theta_{1} \cdot \nabla_{\gamma} \psi_{1}\right| \\
& \quad \leq C \kappa_{1}\left(\frac{|y|}{|x|^{2}|z|}\left|\partial_{x} \theta_{1}^{0} \tilde{\theta}_{1}^{1} \tilde{\theta}^{3}+\theta_{1}^{0} \partial_{x} \tilde{\theta}_{1}^{1} \tilde{\theta}^{3}\right|+\frac{1}{|x||z|}\left|\partial_{y} \theta_{1}^{0} \tilde{\theta}_{1}^{1} \tilde{\theta}^{3}\right|+\frac{|y|}{|x||z|^{2}}\left|\theta_{1}^{0} \partial_{z} \tilde{\theta}_{1}^{1} \tilde{\theta}^{3}+\theta_{1}^{0} \tilde{\theta}_{1}^{1} \partial_{z} \tilde{\theta}^{3}\right|\right) \\
& \quad \leq C \kappa_{1}\left(\frac{R_{0}^{\frac{1}{2}}}{R_{1}^{2}|z|^{\frac{1}{3}}}\left|\partial_{x} \theta_{1}^{0} \tilde{\theta}_{1}^{1} \tilde{\theta}^{3}+\theta_{1}^{0} \partial_{x} \tilde{\theta}_{1}^{1} \tilde{\theta}^{3}\right|+\frac{1}{R_{1}|z|^{\frac{2}{3}}}\left|\partial_{y} \theta_{1}^{0} \tilde{\theta}_{1}^{1} \tilde{\theta}^{3}\right|+\frac{R_{0}^{\frac{1}{2}}}{R_{1}|z|^{\frac{5}{3}}}\left|\theta_{1}^{0} \partial_{z} \tilde{\theta}_{1}^{1} \tilde{\theta}^{3}+\theta_{1}^{0} \tilde{\theta}_{1}^{1} \partial_{z} \tilde{\theta}^{3}\right|\right) \\
& \quad \leq C \kappa_{1}\left(\frac{R_{0}^{\frac{1}{2}}}{R_{1}^{4}}+K_{R_{3}}\right) .
\end{aligned}
$$

We next estimate the cut-off terms involving $\psi_{2}$. Similar to (4.25), we can write $\psi_{2} \mathcal{M}\left(\theta_{2}\right)$ as

$$
\begin{aligned}
\psi_{2} \mathcal{M}\left(\theta_{2}\right)= & \sigma(y-x) \psi_{2}\left(\partial_{x} \theta_{2}^{2} \theta_{2}^{1} \tilde{\theta}^{3}+\theta_{2}^{2} \partial_{x} \theta_{2}^{1} \tilde{\theta}^{3}\right)-(x z+y) \psi_{2} \partial_{y} \theta_{2}^{2} \theta_{2}^{1} \tilde{\theta}^{3}+x y \psi_{1}\left(\theta_{2}^{2} \partial_{z} \theta_{2}^{1} \tilde{\theta}^{3}+\theta_{2}^{2} \theta_{2}^{1} \partial_{z} \tilde{\theta}^{3}\right) \\
& +\gamma_{1} \psi_{2}\left(\partial_{x}^{2} \theta_{2}^{2} \theta_{2}^{1} \tilde{\theta}^{3}+\theta_{2}^{2} \partial_{x}^{2} \theta_{2}^{1} \tilde{\theta}^{3}+2 \partial_{x} \theta_{2}^{2} \partial_{x} \theta_{2}^{1} \tilde{\theta}^{3}\right)+\gamma_{2} \psi_{2} \partial_{y}^{2} \theta_{2}^{2} \theta_{2}^{1} \tilde{\theta}^{3}+ \\
& +\gamma_{3} \psi_{2}\left(\theta_{2}^{2} \partial_{z}^{2} \theta_{2}^{1} \tilde{\theta}^{3}+\theta_{2}^{2} \theta_{2}^{1} \partial_{z}^{2} \tilde{\theta}^{3}+2 \theta_{2} \partial_{z} \theta_{2}^{1} \partial_{z} \tilde{\theta}^{3}\right) .
\end{aligned}
$$

Due to the presence of $\theta_{2}^{1}$ and/or its derivatives, each term in (4.29) is supported on the set $\left\{|x||z|^{\frac{1}{3}} \leq\right.$ $\left.2 R_{1}\right\}$, and therefore the estimate (4.14) applies. Similar to the above, the only terms that cannot be estimated by $K_{R_{3}}$ are $x z \psi_{2} \partial_{y} \theta_{2}^{2} \theta_{2}^{1} \tilde{\theta}^{3}$ and $\gamma_{1} \psi_{2} \theta_{2}^{2} \partial_{x}^{2} \theta_{2}^{1} \tilde{\theta}^{3}$, and for those we have

$$
\left|x z \psi_{2} \partial_{y} \theta_{2}^{2} \theta_{2}^{1} \tilde{\theta}^{3}\right| \leq C \kappa_{2} \frac{|x||z| R_{1}^{2}|y|}{|z|^{2 / 3} R_{2}} \leq C \kappa_{2} \frac{R_{1}^{3}}{R_{2}^{1 / 2}}
$$

and, by definition of $\theta_{1}$,

$$
\begin{aligned}
\left|\gamma_{1} \psi_{2} \theta_{2}^{2} \partial_{x}^{2} \theta_{2}^{1} \tilde{\theta}^{3}\right| & \leq C \kappa_{2} \tilde{\theta}^{3} \mathbb{1}_{x^{2}+y^{2} \leq 2 R_{2}, R_{1} \leq|x||z|^{1 / 3} \leq 2 R_{1}} \\
& \leq C \kappa_{2} \tilde{\theta}^{3} \mathbb{1}_{x^{2}+y^{2} \leq R_{0}, R_{1} \leq|x||z|^{1 / 3} \leq 2 R_{1}}+C \kappa_{2} \mathbb{1}_{\mathcal{R}_{0}} \\
& \leq C \kappa_{2} \theta_{1}+C \kappa_{2} \mathbb{1}_{\mathcal{R}_{0}} .
\end{aligned}
$$


Hence, we have

$$
\left|\psi_{2} \mathcal{M}\left(\theta_{2}\right)\right| \leq C \kappa_{2}\left(\frac{R_{1}^{3}}{R_{2}^{1 / 2}}+\theta_{1}+\mathbb{1}_{\mathcal{R}_{0}}+K_{R_{3}}\right)
$$

where $K_{R_{3}}$ is as in (4.24).

After expanding $\nabla_{\gamma} \theta_{2} \cdot \nabla_{\gamma} \psi_{2}$, the only terms that cannot be bounded by $K_{R_{3}}$ are $\gamma_{1} \partial_{x} \psi_{2} \partial_{x} \theta_{2}^{2} \theta_{2}^{1} \tilde{\theta}_{2}^{3}$ and $\gamma_{1} \partial_{x} \psi_{2} \theta_{2}^{2} \partial_{x} \theta_{2}^{1} \tilde{\theta}_{2}^{3}$. However, if $R_{2} \geq R_{0}$

$$
\left|\gamma_{1} \partial_{x} \psi_{2} \partial_{x} \theta_{2}^{2} \theta_{2}^{1} \tilde{\theta}^{3}\right| \leq C \kappa_{2} \frac{|x y|}{R_{2}} \mathbb{1}_{x^{2}+y^{2} \leq 2 R_{2}} \leq C \kappa_{2} \mathbb{1}_{R_{2} \leq x^{2}+y^{2} \leq 2 R_{2}} \leq C \kappa_{2} \mathbb{1}_{\mathcal{R}_{0}}
$$

and on $\mathcal{R}_{2}$

$$
\left|\gamma_{1} \partial_{x} \psi_{2} \theta_{2}^{2} \partial_{x} \theta_{2}^{1} \tilde{\theta}^{3}\right| \leq C \kappa_{2} \frac{|x y||z|^{\frac{1}{3}}}{R_{2} R_{1}} \mathbb{1}_{x^{2}+y^{2} \leq 2 R_{2},|x||z|^{\frac{1}{3}} \leq 2 R_{1}} \leq C \frac{\kappa_{2}}{R_{2}^{\frac{1}{2}}}
$$

Overall,

$$
\left|\nabla_{\gamma} \theta_{2} \cdot \nabla_{\gamma} \psi_{2}\right| \leq C \kappa_{2}\left(\mathbb{1}_{\mathcal{R}_{0}}+\frac{1}{R_{2}^{\frac{1}{2}}}+K_{R_{3}}\right) .
$$

Let us now gather the estimates (4.5), (4.11), (4.15), (4.27), (4.28), (4.30), and (4.31) to obtain for $R_{2} \geq R_{0}$

$$
\begin{aligned}
\mathcal{M}(V) \leq & -2 \sigma x^{2}-2 y^{2}+\bar{\gamma}-\kappa_{1} \theta_{1}\left(1-\frac{C R_{0}^{3}}{R_{1}}\right)-\kappa_{2} \theta_{2}\left(1-\frac{C R_{1}^{2} R_{2}}{R_{3}^{1 / 3}}\right) \\
& +C\left(\kappa_{1}+\kappa_{2}\right) \mathbb{1}_{\mathcal{R}_{0}}+C \kappa_{1}\left(\frac{1}{R_{1}}+\frac{R_{0}^{\frac{1}{2}}}{R_{1}^{4}}\right)+C \kappa_{2} \frac{R_{1}^{3}}{R_{2}^{1 / 2}}+C \kappa_{2} \theta_{1}+K_{R_{3}}\left(\kappa_{1}+\kappa_{2}\right),
\end{aligned}
$$

where $\bar{\gamma}:=2\left(\gamma_{1}+\gamma_{2}+\gamma_{3}\right)$. Let us fix $\kappa_{2}=16 \bar{\gamma}, \kappa_{1}$ such that $\frac{\kappa_{1}}{2} \geq \max \left\{4 \bar{\gamma}, C \kappa_{2}\right\}$ and $R_{0} \geq 1$ such that

$$
\left(2 \sigma x^{2}+y^{2}\right) \geq 4 \bar{\gamma}+C\left(\kappa_{1}+\kappa_{2}\right) \quad \text { in } \mathcal{R}_{0} .
$$

Then, choose $R_{1}$ such that

$$
C \kappa_{1}\left(\frac{1}{R_{1}}+\frac{R_{0}^{\frac{1}{2}}}{R_{1}^{4}}\right) \leq \frac{\bar{\gamma}}{3} \quad \text { and } \quad \frac{C R_{0}^{3}}{R_{1}} \leq \frac{1}{2}
$$

and $R_{2} \geq R_{0}$ such that

$$
C \kappa_{2} \frac{R_{1}^{3}}{R_{2}^{1 / 2}} \leq \frac{\bar{\gamma}}{3}
$$

Finally, choose $R_{3}$ such that

$$
K_{R_{3}}\left(\kappa_{1}+\kappa_{2}\right) \leq \frac{\bar{\gamma}}{3} \quad \text { and } \quad \frac{C R_{1}^{2} R_{2}}{R_{3}^{1 / 3}} \leq \frac{1}{4} .
$$

With these parameter selections and referring back to (4.18), (4.19) we therefore have

$$
\mathcal{M}(V) \leq-4 \bar{\gamma} \mathbb{1}_{\mathcal{R}_{0}}+2 \bar{\gamma}-\frac{\kappa_{1}}{2} \mathbb{1}_{\mathcal{R}_{1}}-\frac{\kappa_{2}}{4} \mathbb{1}_{\mathcal{R}_{2}} \leq-2 \bar{\gamma}+4 \bar{\gamma}\left(1-\mathbb{1}_{\mathcal{R}_{0} \cup \mathcal{R}_{1} \cup \mathcal{R}_{2}}\right)
$$

Since $R_{1} \leq R_{2}$, one has $\left\{x^{2}+y^{2} \leq R_{0},|z| \geq R_{3}\right\} \subset \mathcal{R}_{1} \cup \mathcal{R}_{2}$, and therefore $\left(1-\mathbb{1}_{\mathcal{R}_{0} \cup \mathcal{R}_{1} \cup \mathcal{R}_{2}}\right)=\mathbb{1}_{\mathcal{K}}$, where $\mathcal{K} \subset\left\{x^{2}+y^{2} \leq R_{0},|z| \leq R_{3}\right\}$ is bounded. Consequently, (4.3) follows with $c=2 \bar{\gamma}$ and $d=4 \bar{\gamma}$. 
Finally let us address the non-negativity of $V$. Notice that our selection of the parameters $R_{0}, R_{1}$, $R_{2}, R_{3}$ and of $\kappa_{1}, \kappa_{2}$ was made independent of the value $\kappa_{0}$ (see (4.20)). Notice however that, by (4.26) we have

$$
\left|\theta_{1} \psi_{1}\right| \leq C \kappa_{1} \frac{R_{1}^{1 / 2}}{R_{1} R_{3}^{2 / 3}}
$$

Similar to (4.14) we observe that

$$
\left|\theta_{2} \psi_{2}\right| \leq C \kappa_{2} \frac{R_{1}^{2}}{R_{3}^{2 / 3}}
$$

Thus having fixed $R_{0}, R_{1}, R_{2}, R_{3}, \kappa_{1}, \kappa_{2}$ and referring back to (4.20) we have

$$
V \geq x^{2}+y^{2}+z^{2}-\sigma^{2}-C \kappa_{1} \frac{R_{1}^{1 / 2}}{R_{1} R_{3}^{2 / 3}} C \kappa_{2} \frac{R_{1}^{2}}{R_{3}^{2 / 3}}+\kappa_{0}
$$

making clear that $\kappa_{0}$ can be selected so that $V$ is positive for every $(x, y, z) \in \mathbb{R}^{3}$. The proof is now complete.

\section{Sensitivity with Respect to Convective Forcing.}

This section addresses some special cases of a very degenerate stochastic forcing when $\beta=0$ in (1.1). First, we establish Theorem 1.2 part (ii) by using the test function $M$ given in (1.6).

5.1. Non-existence under highly degenerate noise. Before proceeding to the rigorous proof of Theorem 1.2, (ii) we present a formal argument. Suppose that we had an invariant probability measure $\mu$ for (1.1) with $\beta=\gamma_{1}=0$. Let us proceed with the unjustified assumption that

$$
\int_{\mathbb{R}^{3}}|X|^{2} \mu(d X)<\infty
$$

Applying Itô's formula to the function $M(x, y, z):=2 \sigma z-x^{2}$, with the process $\left(x_{t}, y_{t}, z_{t}\right)$ initially distributed according to such an invariant measure $\mu$, we obtain

$$
\mathbb{E}_{\mu}\left[2 \sigma z_{t}-x_{t}^{2}\right]=\mathbb{E}_{\mu}\left[2 \sigma z_{0}-x_{0}^{2}\right]+\mathbb{E}_{\mu} \int_{0}^{t}[2 \sigma x y-2 x \sigma(y-x)] d s .
$$

Thus, stationarity and simple algebraic manipulations, cf. (1.7), imply that

$$
\mathbb{E}_{\mu} \int_{0}^{t} x^{2} d s=0
$$

so that $x_{t} \equiv 0$ for every $t \geq 0$.

Now we address two cases. First, we suppose that that $\gamma_{3}>0$. In this situation we apply Itô's lemma to $z^{2}$ and use that $x_{t} \equiv 0$ to find $d z^{2}=2 \gamma_{3} d t+2 \sqrt{2 \gamma_{3}} z d B_{2}$. Integrating and taking expectations we obtain

$$
\mathbb{E}_{\mu} z_{t}^{2}=\mathbb{E}_{\mu} z_{0}^{2}+2 \gamma_{3}
$$

which contradicts stationarity if $\gamma_{3}>0$.

Now let us consider the second case when $\gamma_{3}=0$ but $\gamma_{2}>0$. In this situation the stationary process $\tilde{X}:=\left(x_{t}, y_{t}\right)$ started with initial conditions distributed according to the first two components of the invariant probability measure $\mu$ which satisfies the first two components of (1.1) maintains

$$
d x=\sigma y d t, \quad x_{0}=0, \quad d y=-y d t+\sqrt{2 \gamma_{2}} d B_{2} .
$$

Here, once again we are using that $x_{t} \equiv 0$ we obtain

$$
\sigma y=\frac{d x}{d t}=0
$$

and therefore $y=0$ a contradiction to $\gamma_{2} \neq 0$. 
To make the above arguments rigorous and avoid the assumption (5.1), we use cut-off functions and carefully pass to a limit. We now provide the details.

Proof of Theorem 1.2, (ii). Let $h:[0,2] \rightarrow \mathbb{R}$ be a non-decreasing $C^{2}$ function such that

$$
h(0)=h^{\prime \prime}(0)=h^{\prime}(2)=h^{\prime \prime}(2)=0, \quad h^{\prime}(0)=1, \quad h(2)=1
$$

and $\max _{[0,2]}\left|h^{\prime}\right| \leq 1$. Denote $c^{*}=\max _{[0,2]}\left|h^{\prime \prime}\right|$. It is easy to see that such a function indeed exists. For each $N \geq 1$, define a $C^{2}$ function $F_{N}: \mathbb{R} \rightarrow \mathbb{R}$ as an odd function with

$$
F_{N}(x)= \begin{cases}x & x \in[0, N], \\ h(x-N)+N & x \in[N, N+2], \\ N+1 & x \geq N+2 .\end{cases}
$$

Note that $F_{N}^{\prime} \geq 0, \max _{[0,2]}\left|F_{N}^{\prime}\right| \leq 1$, and $\max _{[0,2]}\left|F_{N}^{\prime \prime}\right|=c^{*}$.

To obtain a contradiction, assume that there is an invariant probability measure $\mu$ of (1.1) and let $(x, y, z)$ have law $\mu$. Since $\mu$ is a probability measure, there exists an increasing sequence of integers $\left(N_{j}\right)_{j=1}^{\infty}$ with $N_{j+1}-N_{j} \geq 2$ such that

$$
\lim _{j \rightarrow \infty} \mathbb{P}\left(\left|2 \sigma z-x^{2}\right| \in\left[N_{j}, N_{j}+2\right]\right)=0 .
$$

If we apply Itô's formula to $F_{N}\left(2 \sigma z-x^{2}\right)$, we obtain

$$
\begin{aligned}
\mathbb{E}_{\mu} F_{N}\left(2 \sigma z_{t}-x_{t}^{2}\right)= & \left.\mathbb{E}_{\mu} F_{N}\left(2 \sigma z_{0}-x_{0}^{2}\right)\right) \\
& +\mathbb{E}_{\mu} \int_{0}^{t}\left(F_{N}^{\prime}\left(2 \sigma z-x^{2}\right)(2 \sigma x y-2 x \sigma(y-x))+F_{N}^{\prime \prime}\left(2 \sigma z-x^{2}\right) 4 \sigma^{2} \gamma_{3}\right) d s .
\end{aligned}
$$

Simple algebraic manipulations and stationarity yield

$$
\mathbb{E}_{\mu} x^{2} F_{N}^{\prime}\left(2 \sigma z_{t}-x_{t}^{2}\right)=-2 \sigma \gamma_{3} \mathbb{E}_{\mu} F_{N}^{\prime \prime}\left(2 \sigma z_{t}-x_{t}^{2}\right) .
$$

Next, we verify that $F_{N_{j+1}}^{\prime} \geq F_{N_{j}}^{\prime}$ for any $j$. Indeed, for $|\xi| \leq N_{j}$ one has $1=F_{N_{j}}^{\prime}(\xi)=F_{N_{j+1}}^{\prime}(\xi)$ and for $|\xi| \geq N_{j+2}$ one has $F_{N_{j}}^{\prime}(\xi)=0 \leq F_{N_{j+1}}^{\prime}(\xi)$. Finally, since $N_{j+1} \geq N_{j}+2$, for any $|\xi| \in\left[N_{j}, N_{j}+2\right]$, we have $F_{N_{j}}^{\prime}(\xi) \leq 1=F_{N_{j+1}}^{\prime}(\xi)$. Thus, $\left(F_{N_{j}}^{\prime}\right)$ is an non-decreasing sequence of non-negative functions that converge pointwise to 1 on $\mathbb{R}$. Therefore, by the monotone convergence theorem and (5.4), we have

$$
\mathbb{E} x^{2}=\lim _{j \rightarrow \infty} \mathbb{E} x^{2} F_{N_{j}}^{\prime}\left(2 \sigma z-x^{2}\right)=-2 \sigma \gamma_{3} \lim _{j \rightarrow \infty} \mathbb{E} F_{N_{j}}^{\prime \prime}\left(2 \sigma z-x^{2}\right) .
$$

Finally, from $\left|F_{N}^{\prime \prime}\right| \leq c^{*}, F_{N}^{\prime \prime}=0$ on the complement of $[N, N+2]$, and (5.3) follows

$$
\lim _{j \rightarrow \infty} \mathbb{E} F_{N_{j}}^{\prime \prime}\left(2 \sigma z-x^{2}\right) \leq c^{*} \mathbb{P}\left(2 \sigma z-x^{2} \in\left[N_{j}, N_{j}+2\right]\right)=0 .
$$

Combining (5.5) and (5.6) yields $\mathbb{E} x^{2}=0$. However, if $\mathbb{E} x^{2}=0$, then, $x=0$ almost surely and by the third equation of the Lorenz system, we have $z(t)=z(0)+\sqrt{2 \gamma_{3}} B_{3}(t)$. This contradicts invariance.

5.2. Uniqueness when the noise component acts only on the convection component of the system. We next turn to the case when $\gamma_{1}>0$ but $\beta=\gamma_{2}=\gamma_{3}=0$. In this special case of Theorem 1.2 (i), we can moreover give an explicit form for the invariant probability measure.

Proposition 5.1. Consider (1.1) with $\sigma>0$ and $\rho \in \mathbb{R}$. If $\gamma_{1}>0, \gamma_{2}=\gamma_{3}=0$, and $\beta=0$, then (4.2) has precisely one statistically invariant state given by the product measure

$$
\mu=\nu_{0, \gamma_{1} / \sigma} \times \delta_{0} \times \delta_{\rho}
$$

where $\delta_{a}$ is the Dirac measure concentrated at a and $\nu_{m, s}$ is the 1-d Guassian measure with mean $m$ and variance $s$ 
Once again, before proceeding to a rigorous proof, we present a formal argument. Suppose that in this parameter range there exists an invariant probability measure $\mu$ of (1.1) and impose the apriori unjustified condition (5.1). Let $(x, y, z)$ be the solution starting for an initial condition distributed as $\mu$. Observe that

$$
\frac{1}{2} \frac{d}{d t}\left(y^{2}+(z-\rho)^{2}\right)=x(\rho-z) y-y^{2}+x y(z-\rho)=-y^{2} .
$$

Integrating this expression in time, taking expected values and using stationarity one finds that $\mathbb{E}_{\mu} \int_{0}^{t} y^{2} d s=0$ so that $y_{t} \equiv 0$ for every $t \geq 0$ by path continuity. Then, as a consequence of this calculation, we infer that $\frac{d}{d t} z=0$ so that $z_{t} \equiv z_{0}$ for every $t \geq 0$. Thus, with stationarity, the equation for $\tilde{X}_{t}=\left(x_{t}, y_{t}\right)$ reduces to

$$
d x=-\sigma x d t+\sqrt{2 \gamma_{1}} d B_{1}, \quad d y=\left(z_{0}-\rho\right) x d t, \quad y_{0}=0 .
$$

The stationarity implies that for every $t, T$

$$
\left(z_{0}-\rho\right) \mathbb{E} \int_{t}^{T} x d s=\mathbb{E} y(T)-\mathbb{E} y(t)=0 .
$$

Since $x$ is almost surely continuous, either $z_{0}=\rho$ or $x=0$. The latter case leads to a immediate contradiction, whereas the former one implies that (5.7) is the only invariant state of (1.1).

Proof of Proposition 5.1. By Theorem 1.2 (i), there exists an invariant probability measure $\mu$, and let $(x, y, z)$ be a random initial condition distributed according to $\mu$. For each $N \geq 1$, let $F_{N}$ be as in (5.2). Similar to the above, fix an increasing sequence $\left(N_{j}\right)_{j=0}^{\infty}$ such that $N_{j+1} \geq N_{j}$. Then, applying Itô's formula to $F_{N}\left(y^{2}+z^{2}\right)$ and taking expected values gives

$$
\mathbb{E} F_{N}\left(y_{t}^{2}+z_{t}^{2}\right)=\mathbb{E} F_{N}\left(y_{0}^{2}+z_{0}^{2}\right)+\mathbb{E} \int_{0}^{t} F_{N}^{\prime}\left(y^{2}+z^{2}\right)(-2 y(x z+y)+2 z x y) d s .
$$

Since the process is stationary, we have

$$
\mathbb{E} F_{N}^{\prime}\left(y^{2}+z^{2}\right) y^{2}=0 .
$$

As in the proof of Theorem 1.2 (ii), by using that $\left(F_{N_{j}}^{\prime}\right)$ is an increasing sequence converging pointwise to 1 , the monotone convergence theorem implies

$$
\mathbb{E} y^{2}=0 .
$$

However, if $y=0$ almost surely, then $z^{\prime}=0$, and therefore $z_{t}=z_{0}$, and $x$ is an invariant state of

$$
d x=-\sigma x d t+\sqrt{2 \gamma_{1}} d B_{1}
$$

as desired.

\section{Non-Existence of Stationary States in the Presence of a Linear Instability}

In this section, we prove Theorem 1.2 part (iii) by constructing functions $V_{i}$ satisfying the hypotheses of Theorem 2.2. In the expressions that follow, we assume that all constants depend implicitly on $\sigma, \beta, \gamma_{1}, \gamma_{2}$, and $\gamma_{3}$. Any other dependence will be indicated explicitly.

6.1. Construction overview. Before proceeding to the proof, let us overview the construction of $V_{1}$ and $V_{2}$ needed to apply Theorem 2.2. We remark that the function $V_{1}$ identifies 'bad' initial conditions from which the dynamics takes too long to return near the origin. Because $\beta<0$, we note that the $z$ process in equation (1.1) grows exponentially fast when it is initially large and when the product $x y$ is not too large. In fact, if one considers the test function

$$
M(x, y, z)=2 \sigma z-x^{2},
$$


then we note that

$$
\mathcal{L} M(x, y, z)=2 \sigma\left(|\beta| z+x^{2}\right)-2 \gamma_{1} .
$$

Hence, if $x^{2}$ is dominated by $z$, then the system (1.1) grows exponentially fast on average. However, we have to be careful because the noise can drive the dynamics out of the region $\left\{x^{2}<|\beta| z\right\}$. To see that such scenario does not occur with high enough probability, we have to modify $M$ and choose appropriate $V_{2}$.

Let us first discuss possible candidates for $V_{2}$. It is easy to check that $\mathcal{L} H$ is neither bounded from above nor from below, and therefore it is not a suitable choice for $V_{2}$. However, we will see that $\mathcal{L}(\ln H)$ is bounded outside of a compact set, and as such we use an appropriate multiple of $\ln H$ for the function $V_{2}$. To satisfy the assumption (p3) in Theorem 2.2, it is necessary that $V_{1}$ has smaller than logarithmic increase at infinity. Given the analysis above, a natural choice would be $F \circ M$, with slowly growing $F$. However, unlike $H, M$ does not have a definite sign, and therefore to define $V_{1}=F \circ M$ one has to define $F$ on the whole real line. We will verify below that $F(\zeta)=\ln \ln \zeta$ indeed produces $\mathcal{L}(F \circ M(x, y, z)) \geq 0$ for large $M(x, y, z)$, but $F$ is not even defined for $M(x, y, z) \leq 0$. In addition, the function $\zeta \mapsto F(|\zeta+C|)$ still does not satisfy the desired inequality. Therefore, we define $F$ to be the double logarithm for large positive values of $\zeta$ and $F \equiv 0$ on $(-\infty, 0)$. The final challenge is to connect these two regions as a smooth function that satisfies $\mathcal{L}(F \circ M) \geq 0$.

6.2. The construction. Based on the heuristics for the construction of $V_{1}$ and $V_{2}$, we now provide a rigorous proof.

Proof of Theorem 1.2 (iii). We define $V_{1}, V_{2}: \mathbb{R}^{3} \rightarrow \mathbb{R}$ satisfying the hypotheses of Theorem 2.2

Step 1. Fix $R>1$ such that $H(x, y, z)>1$ for any $|(x, y, z)|>R$. Let $W_{2} \in C^{2}\left(\mathbb{R}^{3}\right)$ satisfy

$$
W_{2}(x, y, z)=\ln H(x, y, z) \quad \text { for } \quad|(x, y, z)|>R .
$$

Then, $W_{2}>0$ outside of a compact set. Moreover, standard calculations yield

$$
\begin{aligned}
\mathcal{L} W_{2}(x, y, z)= & \frac{2|\beta| z^{2}-2 y^{2}-2 \sigma x^{2}-2|\beta|(\sigma+\rho) z+2\left(\gamma_{1}+\gamma_{2}+\gamma_{3}\right)}{H(x, y, z)} \\
& -\frac{4\left(x^{2} \gamma_{1}+y^{2} \gamma_{2}+(z-(\rho+\sigma))^{2} \gamma_{3}\right)}{H^{2}(x, y, z)} .
\end{aligned}
$$

Consequently, there exists a constant $K>0$ such that

$$
\mathcal{L} W_{2}(x, y, z) \leq K \text { for all }(x, y, z) \in \mathbb{R}^{3} .
$$

We thus define $V_{2}=W_{2} / K$.

Step 2. Define constants

$$
A=\frac{2 \gamma_{1}+2}{|\beta|}, \quad m=\max \left\{2 \gamma_{1}, 2 \sigma^{2} \gamma_{3}\right\}
$$

and let

$$
f(\zeta):=(1-\cos \zeta)^{2}
$$

One can check that $f(0)=f^{\prime}(0)=f^{\prime \prime}(0)=0$ and $f$ is (strictly) increasing on $(0, \pi)$, convex on $\left(0, \frac{2}{3} \pi\right)$ and concave on $\left(\frac{2}{3} \pi, \pi\right)$. In particular $f^{\prime}\left(\frac{2}{3} \pi\right)>0=f^{\prime \prime}\left(\frac{2}{3} \pi\right)$. By continuity, fix $B>\frac{2}{3} \pi$ close to $\frac{2}{3} \pi$ such that $f^{\prime} \geq-m f^{\prime \prime}$ on $\left(\frac{2}{3} \pi, B\right)$.

Next, for constants $c_{0}, c_{1}, c_{2}$ to be determined in a moment, define

$$
\Psi(\zeta)= \begin{cases}0 & \zeta<0, \\ (1-\cos \zeta)^{2}=f(\zeta) & \zeta \in[0, B], \\ c_{0} \ln \ln \left(\zeta+c_{1}\right)+c_{2} & \zeta>B .\end{cases}
$$


We now claim that $c_{0}, c_{1}, c_{2}$ can be chosen such that $\Psi$ is $C^{2}$ function. Because $\Psi$ is $C^{2}$ function at 0 , we have left to show that we can find $c_{0}, c_{1}, c_{2}$ such that

$$
\begin{aligned}
c_{0} \ln \ln \left(B+c_{1}\right)+c_{2} & =f(B)>0, \\
\frac{c_{0}}{\left(B+c_{1}\right) \ln \left(B+c_{1}\right)} & =f^{\prime}(B)>0, \\
-\frac{c_{0}\left(1+\ln \left(B+c_{1}\right)\right)}{\left[\left(B+c_{1}\right) \ln \left(B+c_{1}\right)\right]^{2}} & =f^{\prime \prime}(B)<0 .
\end{aligned}
$$

Substituting the second equation into the third one, we obtain

$$
\frac{1+\ln \left(B+c_{1}\right)}{\left(B+c_{1}\right) \ln \left(B+c_{1}\right)}=-\frac{f^{\prime \prime}(B)}{f^{\prime}(B)}>0 .
$$

However, the function

$$
z \mapsto \frac{1+\ln z}{z \ln z}
$$

is positive and decreasing on $(1, \infty)$ with a vertical asymptote at $z=1$ and decaying at infinity. Thus, there exists (unique) $c_{1}$ such that $B+c_{1}>1$ and (6.1) holds true. Then, for already fixed $c_{1}$ we set

$$
c_{0}=f^{\prime}(B)\left(B+c_{1}\right) \ln \left(B+c_{1}\right)>0
$$

and

$$
c_{2}=f(B)-c_{0} \ln \ln \left(B+c_{1}\right) .
$$

It now follows that $\Psi$ is $C^{2}$ with this choice of constants $c_{0}, c_{1}, c_{2}$.

Finally fix $\lambda \in(0,1)$ such that

$$
1 \geq \lambda m \frac{\left(1+\ln \left(B+c_{1}\right)\right)}{\left(B+c_{1}\right) \ln \left(B+c_{1}\right)}
$$

and define $V_{1}$ by

$$
V_{1}(x, y, z)=\Psi\left(\lambda\left(2 \sigma z-x^{2}-A\right)\right)
$$

and note that $V_{1}$ is $C^{2}$ function and

$$
\mathcal{L} V_{1}=\left(2 \sigma|\beta| z+2 x^{2}-2 \gamma_{1}\right) \lambda \Psi^{\prime}+\left(4 x^{2} \gamma_{1}+4 \sigma^{2} \gamma_{3}\right) \lambda^{2} \Psi^{\prime \prime}
$$

where, for clarity of presentation, we omitted the argument $(x, y, z)$ of $V_{1}$, and $\zeta:=\lambda\left(2 \sigma z-x^{2}-A\right)$ of $\Psi$.

Step 3. We claim that

$$
\mathcal{L} V_{1} \geq 0
$$

First, if $\zeta \leq 0$, then $\Psi^{\prime}=\Psi^{\prime \prime}=0$ and (6.3) follows. For the case when $\zeta \geq 0$, note that since $A=\frac{2 \gamma_{1}+2}{|\beta|}$, $\zeta=\lambda\left(2 \sigma z-x^{2}-A\right) \geq 0$ implies

$$
2 \sigma z \geq 2 \sigma z-x^{2} \geq A=\frac{2 \gamma_{1}+2}{|\beta|},
$$

and consequently $2 \sigma|\beta| z-2 \gamma_{1} \geq 2$. Hence,

$$
2 \sigma|\beta| z+2 x^{2}-2 \gamma_{1} \geq 2\left(x^{2}+1\right), \quad 0 \leq\left(4 x^{2} \gamma_{1}+4 \sigma^{2} \gamma_{3}\right) \leq 2 m\left(x^{2}+1\right) .
$$

Hence, if $\zeta \geq 0$, the coefficients of $\Psi^{\prime}, \Psi^{\prime \prime}$ in (6.2) are non-negative. We split the domain $\zeta \geq 0$ into three pieces and then finally conclude (6.3).

If $\zeta \in\left[0, \frac{2}{3} \pi\right]$, then $\Psi^{\prime}(\zeta), \Psi^{\prime \prime}(\zeta) \geq 0$, and the non-negativity of coefficients of $\Psi^{\prime}, \Psi^{\prime \prime}$ in (6.2) implies $(6.3)$. 
If $\zeta \in\left(\frac{2}{3} \pi, B\right)$, then $\Psi^{\prime}(\zeta)>0$ and $\Psi^{\prime \prime}(\zeta)<0$. Thus, from (6.3) and (6.4) follows

$$
\begin{aligned}
\frac{1}{\lambda} \mathcal{L} V_{1} & \geq\left(2 \sigma|\beta| z+2 x^{2}-2 \gamma_{1}\right) \Psi^{\prime}+\lambda\left(4 x^{2} \gamma_{1}+4 \sigma^{2} \gamma_{3}\right) \Psi^{\prime \prime} \\
& \geq 2\left(x^{2}+1\right) \Psi^{\prime}+2 \lambda m\left(x^{2}+1\right) \Psi^{\prime \prime} \geq 0,
\end{aligned}
$$

where in the last inequality we used the definition of $B$ and $\lambda \in(0,1]$.

Finally, if $\zeta \in[B, \infty)$, then $\Psi(\zeta)=c_{0} \ln \ln \left(\zeta+c_{1}\right)+c_{2}$. Since $c_{0}>0$, one has $\Psi^{\prime}(\zeta)>0, \Psi^{\prime \prime}(\zeta)<0$. Using (6.5) and the fact that the function $z \mapsto \frac{1+z}{z \ln z}$ decreases, we obtain for any $\zeta>B$

$$
\begin{aligned}
\frac{1}{\lambda} \mathcal{L} V_{1} & \geq 2\left(x^{2}+1\right) \Psi^{\prime}+2 \lambda m\left(x^{2}+1\right) \Psi^{\prime \prime} \\
& \geq \frac{2 c_{0}\left(x^{2}+1\right)}{\left(2 \zeta+c_{1}\right) \ln \left(\zeta+c_{1}\right)}\left(1-\lambda m \frac{\left(1+\ln \left(\zeta+c_{1}\right)\right)}{\left(\zeta+c_{1}\right) \ln \left(\zeta+c_{1}\right)}\right) \\
& \geq \frac{2 c_{0}\left(x^{2}+1\right)}{\left(2 \zeta+c_{1}\right) \ln \left(\zeta+c_{1}\right)}\left(1-\lambda m \frac{\left(1+\ln \left(B+c_{1}\right)\right)}{\left(B+c_{1}\right) \ln \left(B+c_{1}\right)}\right) \geq 0,
\end{aligned}
$$

where in the last estimate we used the definition of $\lambda$. Thus, $\mathcal{L} W_{1} \geq 0$ as desired.

Step 4. Let us verify that the assumptions of Theorem 2.2 are satisfied with $V_{1}$ and $V_{2}$. First (p4) follows from the construction. To verify (p1), observe that

$$
\begin{aligned}
\limsup _{|(x, y, z)| \rightarrow \infty} V_{1}(x, y, z) & \geq \lim _{z \rightarrow \infty} V_{1}(0,0, z)=\lim _{z \rightarrow \infty} \Psi(\lambda(2 \sigma z-A)) \\
& =\lim _{z \rightarrow \infty} c_{0} \ln \ln \left(\lambda(2 \sigma z-A)+c_{1}\right)+c_{2}=\infty .
\end{aligned}
$$

Also, $\lim _{|(x, y, z)| \rightarrow \infty} H(x, y, z)=\infty$ and (p2) is satisfied. Finally, (p3) follows from

$$
\begin{aligned}
\limsup _{R \rightarrow \infty} \frac{\sup _{|x, y, z|=R} V_{1}(x, y, z)}{\inf _{|x, y, z|=R} V_{2}(x, y, z)} & \leq \limsup _{R \rightarrow \infty} \frac{V_{1}(0,0, R)}{\ln \left(R^{2}-2(\sigma+\rho) R\right)} \\
& \leq \lim _{R \rightarrow \infty} \frac{c_{0} \ln \ln \left(\lambda(2 \sigma R-A)+c_{1}\right)+c_{2}}{\ln \left(R^{2}-2(\sigma+\rho) R\right)}=0,
\end{aligned}
$$

where we used that $z \mapsto V_{1}(x, y, z)$ is increasing for large $z$ and $(x, y) \mapsto V_{1}(x, y, z)$ is non-increasing. This finishes the proof.

\section{ACKnowledgements}

We would like to thank Centra International de Rencontres Mathmatiques (CIRM), in Marseille, France and the Centro Internazionale per la Ricerca Matematica (CIRM) in Trento, Itally for supporting the first two authors with two short visiting research fellowships where much of this work was conceived. We would also like to acknowledge our colleagues Jonathan Mattingly and Jared Whitehead for helpful feedback on this work.

Our efforts were partially supported under grants NSF-DMS-1816408 (JF), DMS-1313272 (NEGH), DMS-1816551 (NEGH), DMS-1612898 (DPH), DMS-1855504 (DPH), from the National Science Foundation as well as the Simons Foundation travel grant 515990 (NEGH).

\section{REFERENCES}

[AKM12] Avanti Athreya, Tiffany Kolba, and Jonathan Mattingly. Propagating Lyapunov functions to prove noiseinduced stabilization. Electronic Journal of Probability, 17, 2012.

[BD12] Katarína Bodová and Charles Doering. Noise-induced statistically stable oscillations in a deterministically divergent nonlinear dynamical system. Communications in Mathematical Sciences, 10(1):137-157, 2012.

[Bel06] Luc Rey Bellet. Ergodic properties of Markov processes. In Open quantum systems II, pages 1-39. Springer, 2006. 
[BHW12] Jeremiah Birrell, David P Herzog, and Jan Wehr. The transition from ergodic to explosive behavior in a family of stochastic differential equations. Stochastic Processes and their Applications, 122(4):1519-1539, 2012.

[CDG ${ }^{+}$06] E Calzavarini, CR Doering, JD Gibbon, D Lohse, A Tanabe, and F Toschi. Exponentially growing solutions in homogeneous Rayleigh-Bénard convection. Physical Review E, 73(3):035301, 2006.

[CEHRB18] Noé Cuneo, Jean-Pierre Eckmann, Martin Hairer, and Luc Rey-Bellet. Non-equilibrium steady states for networks of oscillators. Electronic Journal of Probability, 23, 2018.

$\left[\mathrm{CFK}^{+} 17\right]$ Joe P Chen, Lance Ford, Derek Kielty, Rajeshwari Majumdar, Heather McCain, Dylan O?Connell, and Fan Ny Shum. Stabilization by noise of a $\mathbb{C}^{2}$-valued coupled system. Stochastics and Dynamics, 17(06):1750046, 2017.

[CZH20] Michele Coti Zelati and Martin Hairer. A noise-induced transition in the lorenz system. arXiv preprint:2004.12815, 2020.

[EH00] J-P Eckmann and Martin Hairer. Non-Equilibrium Statistical Mechanics of Strongly Anharmonic Chains of Oscillators. Communications in Mathematical Physics, 212(1):105-164, 2000.

[EHS17] Elgindi Tarek, Hu Wenqing, and Sverák Vladimír. On 2d Incompressible Euler Equations with Partial Damping. Communications in Mathematical Physics, 355(1):145-159, 2017.

[GHW11] Krzysztof Gawedzki, David P Herzog, and Jan Wehr. Ergodic properties of a model for turbulent dispersion of inertial particles. Communications in mathematical physics, 308(1):49, 2011.

[Hai09] Martin Hairer. How hot can a heat bath get? Communications in Mathematical Physics, 292(1):131-177, 2009 .

[HM09] Martin Hairer and Jonathan C Mattingly. Slow energy dissipation in anharmonic oscillator chains. Communications on Pure and Applied Mathematics: A Journal Issued by the Courant Institute of Mathematical Sciences, 62(8):999-1032, 2009.

[HM15a] D. Herzog and J. Mattingly. Noise-induced stabilization of planar flows I. Electronic Journal of Probability, 20, 2015.

[HM15b] David P Herzog and Jonathan C Mattingly. A practical criterion for positivity of transition densities. Nonlinearity, 28(8):2823, 2015.

[Hör67a] Lars Hörmander. Hypoelliptic second order differential equations. Acta Mathematica, 119:147-171, 1967.

[Hör67b] Lars Hörmander. Hypoelliptic second order differential equations. Acta Mathematica, 119(1):147-171, 1967.

[HP90a] David W Hughes and Michael RE Proctor. Chaos and the effect of noise in a model of three-wave mode coupling. Physica D: Nonlinear Phenomena, 46(2):163-176, 1990.

[HP90b] DW Hughes and MRE Proctor. A low-order model of the shear instability of convection: chaos and the effect of noise. Nonlinearity, 3(1):127, 1990.

$\left[\mathrm{K}^{+} 87\right] \quad$ Wolfgang Kliemann et al. Recurrence and invariant measures for degenerate diffusions. The annals of probability, 15(2):690-707, 1987.

[KCSW19] Tiffany Kolba, Anthony Coniglio, Sarah Sparks, and Daniel Weithers. Noise-Induced Stabilization of Perturbed Hamiltonian Systems. The American Mathematical Monthly, 126(6):505-518, 2019.

[Kha60] Rafail Z Khasminskii. Ergodic properties of recurrent diffusion processes and stabilization of the solution to the Cauchy problem for parabolic equations. Theory of Probability \& Its Applications, 5(2):179-196, 1960.

[Kha11] Rafail Khasminskii. Stochastic stability of differential equations, volume 66. Springer Science \& Business Media, 2011.

[Lor63] Edward N Lorenz. Deterministic nonperiodic flow. Journal of the atmospheric sciences, 20(2):130-141, 1963.

[LS17] Matti Leimbach and Michael Scheutzow. Blow-up of a stable stochastic differential equation. Journal of Dynamics and Differential Equations, 29(2):345-353, 2017.

[MSH02] Jonathan C Mattingly, Andrew M Stuart, and Desmond J Higham. Ergodicity for SDEs and approximations: locally Lipschitz vector fields and degenerate noise. Stochastic processes and their applications, 101(2):185232, 2002.

[MT57] Gisirô Maruyama and Hiroshi Tanaka. Some properties of one-dimensional diffusion processes. Memoirs of the Faculty of Science, Kyushu University. Series A, Mathematics, 11(2):117-141, 1957.

[MT93] Sean P Meyn and Richard L Tweedie. Stability of Markovian processes III: Foster-Lyapunov criteria for continuous-time processes. Advances in Applied Probability, 25(3):518-548, 1993.

[MT12] Sean P Meyn and Richard L Tweedie. Markov chains and stochastic stability. Springer Science \& Business Media, 2012.

[RBT02] Luc Rey-Bellet and Lawrence E Thomas. Exponential convergence to non-equilibrium stationary states in classical statistical mechanics. Communications in mathematical physics, 225(2):305-329, 2002.

[Sch93] Michael Scheutzow. Stabilization and destabilization by noise in the plane. Stochastic Analysis and Applications, 11(1):97-113, 1993.

[SS17] Janet D Scheel and Jörg Schumacher. Predicting transition ranges to fully turbulent viscous boundary layers in low Prandtl number convection flows. Physical Review Fluids, 2(12):123501, 2017. 
[SV72a] D Stroock and SRS Varadhan. On degenerate elliptic-parabolic operators of second order and their associated diffusions. Communications on Pure and Applied Mathematics, 25(6):651-713, 1972.

[SV72b] Daniel W Stroock and Srinivasa RS Varadhan. On the support of diffusion processes with applications to the strong maximum principle. In Proceedings of the Sixth Berkeley Symposium on Mathematical Statistics and Probability (Univ. California, Berkeley, Calif., 1970/1971), volume 3, pages 333-359, 1972.

[Thu92] Olivier Thual. Zero-Prandtl-number convection. Journal of Fluid Mechanics, 240:229-258, 1992.

[Won66] WM Wonham. Liapunov criteria for weak stochastic stability. Journal of Differential Equations, 2(2):195207, 1966 .

\section{Juraj Földes}

Department of Mathematics

University of Virginia

Web: http://www.people.virginia.edu/ jf8dc/

Email: foldes@virginia.edu

\author{
Nathan E. Glatt-Holtz \\ Department of Mathematics \\ Tulane University \\ Web: http://www.math.tulane.edu/ negh/ \\ Email: negh@tulane.edu
}

\author{
David P. Herzog \\ Department of Mathematics \\ Iowa State University \\ Web: http://orion.math.iastate.edu/dherzog/ \\ Email: dherzog@iastate.edu
}

